\title{
Real-time solution of linear computational problems using databases of parametric reduced-order models with arbitrary underlying meshes
}

\author{
David Amsallemª, Radek Tezaura ${ }^{\mathrm{a}}$, Charbel Farhat ${ }^{\mathrm{b}}$ \\ ${ }^{a}$ Department of Aeronautics and Astronautics, Durand Building, 496 Lomita Mall. Stanford University, Stanford, CA \\ 94305-4035, USA \\ ${ }^{b}$ Department of Aeronautics and Astronautics, Institute for Computational and Mathematical Engineering, Department of \\ Mechanical Engineering, Durand Building, 496 Lomita Mall. Stanford University, Stanford, CA 94305-4035, USA
}

\begin{abstract}
A comprehensive approach for real-time computations using a database of parametric, linear, projectionbased reduced-order models (ROMs) based on arbitrary underlying meshes is proposed. In the offline phase of this approach, the parameter space is sampled and linear ROMs defined by linear reduced operators are pre-computed at the sampled parameter points and stored. Then, these operators and associated ROMs are transformed into counterparts that satisfy a certain notion of consistency. In the online phase of this approach, a linear ROM is constructed in real-time at a queried but unsampled parameter point by interpolating the pre-computed linear reduced operators on matrix manifolds and therefore computing an interpolated linear ROM. The proposed overall model reduction framework is illustrated with two applications: a parametric inverse acoustic scattering problem associated with a mockup submarine, and a parametric flutter prediction problem associated with a wing-tank system. The second application is implemented on a mobile device, illustrating the capability of the proposed computational framework to operate in real-time.
\end{abstract}

Keywords: acoustic scattering, aeroelasticity, database of ROMs, interpolation, matrix manifold, mobile computing, parametric model order reduction, real-time computing, reduced-order model

\section{Introduction}

Many engineering applications require the ability to predict the behavior of physical systems in real-time. Among these, one can mention design optimization, optimal control, the solution of inverse problems, as well as uncertainty quantification. All of these applications typically incur a large number of numerical predictions for varying values of operating conditions. Usually, these are described by a set of parameters, and may define boundary conditions, initial conditions, and physical or shape parameters that in turn define the problem of interest and its underlying differential equations. Each of these predictions usually requires computationally intensive computations, as accurate discretizations of the underlying differential equations often lead to large-scale systems of equations.

Projection-based model order reduction (MOR) [1,2] reduces the large computational cost of a simulation performed using a high-dimensional model (HDM) by reducing the number of degrees of freedom in the computation. It achieves this objective by constructing first a reduced-order basis (ROB), then approximating the solution in the subspace described by this ROB. Currently, the most challenging problems faced by MOR are those associated with nonlinearities and parameter variations. For nonlinear systems, MOR requires addressing critical issues pertaining to solution discontinuities when these arise [3] (or regime changes

Email addresses: amsallem@stanford.edu (David Amsallem), rtezaur@stanford.edu (Radek Tezaur), cfarhat@stanford.edu (Charbel Farhat)

$U R L:$ stanford.edu/ amsallem (David Amsallem) 
in general), in order to achieve accuracy at low dimensionality. It also calls for an additional level of approximation to enable translating dimensionality reduction into large computational speedup $[4,5,6,7,8,9]$. Most importantly, the MOR of a parametric nonlinear system is also challenging due to the lack of robustness of a reduced-order model (ROM) with respect to parameter variations, which requires an appropriate training offline $[10,11,12,13]$.

For parametric systems, MOR requires addressing important issues pertaining to the robustness with respect to parameter variations of both the construction process of a reduced-order model (ROM) and the performance of the resulting ROM. In this paper, the focus is set on addressing the latter issues in the context of linear systems. Specifically, the addressed challenge is that of designing a MOR reduction approach for a parametric linear system that is feasible, practical, and yet delivers a real-time capability for performing numerical predictions independently from the complexity of the associated application.

For parametric linear systems, ROM database approaches where reduced linear operators - and associated ROMs - of a common low dimension are pre-computed offline for sampled values of the parameters and stored have been recently developed $[12,14,13,15,16,17]$. In general, the idea is that the stored reduced linear operators can then be interpolated during an online phase of computations to construct in real-time linear ROMs at unsampled parameter values, and use these ROMs to perform real-time predictions on-the-fly.

Following the ROM database approach outlined above, special attention is paid in this paper to the interpolation step. To this effect, it is noted that important mathematical properties of reduced linear operators can be preserved when interpolation is performed on appropriate matrix manifolds $[12,14,15,17]$. Specifically, after appropriately mapping the linear reduced operators, interpolation can be carried out in the tangent space to a relevant matrix manifold. As long as the interpolation procedure preserves the tangent space, the interpolated quantity will also belong to the tangent space and can be mapped back to the manifold, leading to a properties-preserving interpolated reduced quantity. Nevertheless, the interpolation of local, linear, reduced operators is a challenge in itself since each linear reduced operator can be written in terms of a distinct set of generalized coordinates corresponding to the local ROBs associated with each local ROM. To address this issue, approaches based on congruent transformations were proposed in $[14,16,17]$, for the case where the underlying HDMs arise from discretizations defined on a common mesh, which can be a rather severe limitation. Hence, these approaches are not applicable to the more frequent case where different HDMs are defined on different meshes. To remove this limitation, this paper introduces a novel approach that is also based on congruent transformations, but that addresses the challenge associated with arbitrary meshes. To this effect, its remainder is organized as follows.

The problem of interest and ROM database concept are formulated in Section 2. The consistency issue for reduced-order operators and its enforcement are discussed in Section 3 for both cases of common and arbitrary underlying meshes. The general approach for interpolating pre-computed consistent reduced operators in a database of linear ROMs is developed in Section 4. Special attention is paid to parameter sampling, data storage, and data exploitation. The proposed overall MOR approach is applied in Section 5 to the reduction of two parametric computational models. The first one is associated with a parametric inverse acoustic scattering problem featuring multiple meshes. The second model is associated with the flutter analysis on a mobile device of an aeroelastic system for flight conditions ranging from the subsonic to the supersonic regime. In both cases, it is shown that the proposed approach successfully enables real-time predictions. Finally, conclusions are given in Section 6.

\section{Problem formulation and solution approach}

In this paper, linear-time invariant parametric (LTIP) systems of one of the following two forms are considered:

1. First-order LTIP systems of the form

$$
\mathbf{E}(\boldsymbol{\mu}) \frac{d \mathbf{w}}{d t}(t)=\mathbf{A}(\boldsymbol{\mu}) \mathbf{w}(t)+\mathbf{B}(\boldsymbol{\mu}) \mathbf{u}(t)
$$


or their formulation in the frequency domain

$$
(j \omega \mathbf{E}(\boldsymbol{\mu})-\mathbf{A}(\boldsymbol{\mu})) \mathbf{w}(\omega)=\mathbf{B}(\boldsymbol{\mu}) \mathbf{u}(\omega),
$$

where $\mathbf{E}$ and $\mathbf{A}$ are square high-dimensional matrices of dimension $N$ acting on the high-dimensional state vector $\mathbf{w} \in \mathbb{R}^{N}, j^{2}=-1, t \geq 0$ denotes time, and $\omega \geq 0$ frequency. The vector $\mathbf{u} \in \mathbb{R}^{N_{i}}$ denotes the input variable of dimension $N_{i} \ll N$ and $\mathbf{B} \in \mathbb{R}^{N \times N_{i}}$. All operators depend on a vector of $N_{\boldsymbol{\mu}}$ parameters $\boldsymbol{\mu} \in \mathcal{D} \subset \mathbb{R}^{N_{\mu}}$.

For both formulations, an output quantity of interest (QoI) $\mathbf{y} \in \mathbb{R}^{N_{o}}$ is defined as

$$
\mathbf{y}=\mathbf{G}(\boldsymbol{\mu}) \mathbf{w}+\mathbf{H}(\boldsymbol{\mu}) \mathbf{u},
$$

with $N_{o} \ll N, \mathbf{G} \in \mathbb{R}^{N_{o} \times N}$ and $\mathbf{H} \in \mathbb{R}^{N_{o} \times N_{i}}$.

Such first-order LTIP systems arise in the context of heat conduction, advection-diffusion and linearized computational fluid dynamics (CFD) applications, to name just a few.

2. Second-order LTIP systems of the form

$$
\mathbf{M}(\boldsymbol{\mu}) \frac{d^{2} \mathbf{w}}{d t^{2}}(t)+\mathbf{C}(\boldsymbol{\mu}) \frac{d \mathbf{w}}{d t}(t)+\mathbf{K}(\boldsymbol{\mu}) \mathbf{w}(t)=\mathbf{B}(\boldsymbol{\mu}) \mathbf{u}(t)
$$

or their equivalent formulation in the frequency domain

$$
\left(-\omega^{2} \mathbf{M}(\boldsymbol{\mu})+j \omega \mathbf{C}(\boldsymbol{\mu})+\mathbf{K}(\boldsymbol{\mu})\right) \mathbf{w}(\omega)=\mathbf{B}(\boldsymbol{\mu}) \mathbf{u}(\omega) .
$$

Here, $\mathbf{M}, \mathbf{C}$ and $\mathbf{K}$ are also parameter-dependent square linear operators of dimension $N$. For both formulations, an output QoI $\mathbf{y}$ can be defined as in (2).

Second-order LTIP systems arise in many contexts, including structural mechanics, circuit simulation and acoustics.

Throughout this paper, the problem of interest is that of the fast computation of the output variable $\mathbf{y}$ for a given value of the parameter vector $\boldsymbol{\mu} \in \mathcal{D}$, using a computational model and associated algorithm whose complexity do not scale with $N$. To address this problem, an approach based on a database of linear projection-based ROMs is considered. This approach proceeds in two steps.

1. In the first step — which is performed offline - $N_{\text {DB }}$ sample parameter values $\left\{\boldsymbol{\mu}_{i}\right\}_{i=1}^{N_{\mathrm{DB}}} \in \mathcal{D} \subset \mathbb{R}^{N_{\boldsymbol{\mu}}}$ are selected, and ROMs are constructed for each sampled parameter value by defining right and left reduced-order bases (ROBs) $\mathbf{V}(\boldsymbol{\mu}) \in \mathbb{R}^{N \times k}$ and $\mathbf{W}(\boldsymbol{\mu}) \in \mathbb{R}^{N \times k}, k \ll N$ and approximating the state $\mathbf{w}$ as $\mathbf{w} \approx \mathbf{V}(\boldsymbol{\mu}) \mathbf{q}$ where $\mathbf{q} \in \mathbb{R}^{k}$ is either solution of a reduced LTIP system in the time or frequency domain, as detailed below. The output equation in terms of the reduced variable $\mathbf{q}$ is then

$$
\mathbf{y}_{r}=\mathbf{G}_{r}(\boldsymbol{\mu}) \mathbf{q}+\mathbf{H}(\boldsymbol{\mu}) \mathbf{u}
$$

with $\mathbf{G}_{r}(\boldsymbol{\mu})=\mathbf{G}(\boldsymbol{\mu}) \mathbf{V}(\boldsymbol{\mu}) \in \mathbb{R}^{N_{o} \times k}$.

(a) For first-order LTIP systems, the time-domain reduced equations are given by

$$
\mathbf{E}_{r}(\boldsymbol{\mu}) \frac{d \mathbf{q}}{d t}(t)=\mathbf{A}_{r}(\boldsymbol{\mu}) \mathbf{q}(t)+\mathbf{B}_{r}(\boldsymbol{\mu}) \mathbf{u}(t)
$$

Alternatively, the frequency-domain reduced equations are given by

$$
\left(j \omega \mathbf{E}_{r}(\boldsymbol{\mu})-\mathbf{A}_{r}(\boldsymbol{\mu})\right) \mathbf{q}(\omega)=\mathbf{B}_{r}(\boldsymbol{\mu}) \mathbf{u}(\omega)
$$

where $\mathbf{E}_{r}(\boldsymbol{\mu})=\mathbf{W}(\boldsymbol{\mu})^{T} \mathbf{E}(\boldsymbol{\mu}) \mathbf{V}(\boldsymbol{\mu}) \in \mathbb{R}^{k \times k}, \mathbf{A}_{r}(\boldsymbol{\mu})=\mathbf{W}(\boldsymbol{\mu})^{T} \mathbf{A}(\boldsymbol{\mu}) \mathbf{V}(\boldsymbol{\mu}) \in \mathbb{R}^{k \times k}$ and $\mathbf{B}_{r}(\boldsymbol{\mu})=$ $\mathbf{W}(\boldsymbol{\mu})^{T} \mathbf{B}(\boldsymbol{\mu}) \in \mathbb{R}^{k \times N_{i}}$. 
(b) For second-order LTIP systems, the time-domain reduced equations are

$$
\mathbf{M}_{r}(\boldsymbol{\mu}) \frac{d^{2} \mathbf{q}}{d t^{2}}(t)+\mathbf{C}_{r}(\boldsymbol{\mu}) \frac{d \mathbf{q}}{d t}(t)+\mathbf{K}_{r}(\boldsymbol{\mu}) \mathbf{q}(t)=\mathbf{B}_{r}(\boldsymbol{\mu}) \mathbf{u}(t),
$$

where $\mathbf{M}_{r}(\boldsymbol{\mu})=\mathbf{W}(\boldsymbol{\mu})^{T} \mathbf{M}(\boldsymbol{\mu}) \mathbf{V}(\boldsymbol{\mu}), \mathbf{C}_{r}(\boldsymbol{\mu})=\mathbf{W}(\boldsymbol{\mu})^{T} \mathbf{C}(\boldsymbol{\mu}) \mathbf{V}(\boldsymbol{\mu})$ and $\mathbf{K}_{r}(\boldsymbol{\mu})=\mathbf{W}(\boldsymbol{\mu})^{T} \mathbf{K}(\boldsymbol{\mu}) \mathbf{V}(\boldsymbol{\mu})$ are square reduced operators of dimension $k$. Alternatively, the frequency-domain reduced equations are given by

$$
\left(-\omega^{2} \mathbf{M}_{r}(\boldsymbol{\mu})+j \omega \mathbf{C}_{r}(\boldsymbol{\mu})+\mathbf{K}_{r}(\boldsymbol{\mu})\right) \mathbf{q}(\omega)=\mathbf{B}_{r}(\boldsymbol{\mu}) \mathbf{u}(\omega) .
$$

There are several model reduction techniques that can be applied to construct the ROBs $\mathbf{W}(\boldsymbol{\mu})$ and $\mathbf{V}(\boldsymbol{\mu})$ for LTI systems. Among those, the most popular are proper orthogonal decomposition (POD) [2, 18], balanced truncation [1] and moment matching [19, 20].

Once the reduced operators are computed for the sampled values of the parameters, these reduced matrices are stored in a database of the form

$$
\mathcal{D B}=\left\{\boldsymbol{\mu}_{i},\left(\mathbf{E}_{r}\left(\boldsymbol{\mu}_{i}\right), \mathbf{A}_{r}\left(\boldsymbol{\mu}_{i}\right), \mathbf{B}_{r}\left(\boldsymbol{\mu}_{i}\right), \mathbf{G}_{r}\left(\boldsymbol{\mu}_{i}\right), \mathbf{H}\left(\boldsymbol{\mu}_{i}\right)\right)\right\}_{i=1}^{N_{\mathrm{DB}}}
$$

for first-order systems, and

$$
\mathcal{D B}=\left\{\boldsymbol{\mu}_{i},\left(\mathbf{M}_{r}\left(\boldsymbol{\mu}_{i}\right), \mathbf{C}_{r}\left(\boldsymbol{\mu}_{i}\right), \mathbf{K}_{r}\left(\boldsymbol{\mu}_{i}\right), \mathbf{B}_{r}\left(\boldsymbol{\mu}_{i}\right), \mathbf{G}_{r}\left(\boldsymbol{\mu}_{i}\right), \mathbf{H}\left(\boldsymbol{\mu}_{i}\right)\right)\right\}_{i=1}^{N_{\mathrm{DB}}}
$$

for second-order systems.

In this work, the left and right ROBs are assumed to have orthonormal columns with respect to a common symmetric positive definite matrix $\mathcal{M}$, that is $\mathbf{W}(\boldsymbol{\mu})^{T} \mathcal{M} \mathbf{W}(\boldsymbol{\mu})=\mathbf{I}_{k}$ and $\mathbf{V}(\boldsymbol{\mu})^{T} \mathcal{M} \mathbf{V}(\boldsymbol{\mu})=$ $\mathbf{I}_{k}$. This property can be easily enforced a posteriori by applying a Gram-Schmidt orthogonalization procedure to the columns of non-orthonormal ROBs or directly in the ROB construction procedure.

2. In the second step - which is performed online - given a queried value $\boldsymbol{\mu}^{\star} \in \mathcal{D}$ of the parameter vector, reduced operators are constructed by interpolating elements of the database $\mathcal{D B}$.

Two technical issues arise however in the online interpolation step:

1. The reduced quantities are not necessarily defined in the same system of reduced coordinates. Indeed, for each parameter vector $\boldsymbol{\mu}_{i}$, the system of reduced coordinates is defined by the local ROBs $\mathbf{V}\left(\boldsymbol{\mu}_{i}\right)$ and $\mathbf{W}\left(\boldsymbol{\mu}_{i}\right)$. In this case, even an appropriate interpolation of the linear reduced operators may result in interpolation quantities that are not consistent with each other. In the remainder of this paper, two ROBs corresponding to different parameter values will be denoted as consistent if their discrepancy originates only from the variation of the subspace they describe and not the choice of basis in the subspace. A comprehensive approach that addresses this issue is proposed in Section 3.

2. The linear operators stored in the database $\mathcal{D B}$ may have properties that should be preserved by interpolation. An approach relying on interpolation on a matrix manifold was proposed in [17] for preserving such properties. It is briefly recalled in Section 4 and extended to the case of HDMs arising from discretizations over different meshes, where state vectors may even be of different dimensions.

\section{Consistent reduced-order models}

\subsection{Concept}

As underlined in the previous section and in [17], the fact that local, linear, reduced operators are in general pre-computed in different sets of generalized coordinates prevents their direct interpolation. In this paper, two approaches are presented to address this issue. Both rely on a congruence transformation of the reduced operators to enforce consistency. They recognize the fact that the choice of local ROBs is not 
unique. Indeed, for a given right $\operatorname{ROB} \mathbf{V}(\boldsymbol{\mu})$, any $\mathrm{ROB}$ of the form $\mathbf{V}(\boldsymbol{\mu}) \mathbf{Q}$ with $\mathbf{Q}^{T} \mathbf{Q}=\mathbf{I}_{k}$ defines an equally valid coordinate representation for the same ROM [17] with $\mathcal{M}$-orthogonal columns. Similarly, for the left ROB $\mathbf{W}(\boldsymbol{\mu})$, any left ROB of the form $\mathbf{W}(\boldsymbol{\mu}) \mathbf{Z}$ with $\mathbf{Z}^{T} \mathbf{Z}=\mathbf{I}_{k}$ defines an equally valid basis.

In turn, for a given first-order LTI ROM $\mathcal{R}=\left(\mathbf{E}_{r}, \mathbf{A}_{r}, \mathbf{B}_{r}, \mathbf{G}_{r}, \mathbf{H}\right)$, an equivalence class of ROMs under left and right multiplications by orthogonal matrices $\mathbf{Z}$ and $\mathbf{Q}$ can be defined as

$$
\mathcal{C}(\mathcal{R})=\left\{\left(\mathbf{Z}^{T} \mathbf{E}_{r} \mathbf{Q}, \mathbf{Z}^{T} \mathbf{A}_{r} \mathbf{Q}, \mathbf{Z}^{T} \mathbf{B}_{r}, \mathbf{G}_{r} \mathbf{Q}, \mathbf{H}\right) \text { such that } \mathbf{Z}^{T} \mathbf{Z}=\mathbf{I}, \mathbf{Q}^{T} \mathbf{Q}=\mathbf{I}\right\} .
$$

Similarly, for a second-order LTI ROM $\mathcal{R}=\left(\mathbf{M}_{r}, \mathbf{C}_{r}, \mathbf{K}_{r}, \mathbf{B}_{r}, \mathbf{G}_{r}, \mathbf{H}\right)$, the equivalence class of ROMs under left and right multiplications by orthogonal matrices $\mathbf{Z}$ and $\mathbf{Q}$ is

$$
\mathcal{C}(\mathcal{R})=\left\{\left(\mathbf{Z}^{T} \mathbf{M}_{r} \mathbf{Q}, \mathbf{Z}^{T} \mathbf{C}_{r} \mathbf{Q}, \mathbf{Z}^{T} \mathbf{K}_{r} \mathbf{Q}, \mathbf{Z}^{T} \mathbf{B}_{r}, \mathbf{G}_{r} \mathbf{Q}, \mathbf{H}\right) \text { such that } \mathbf{Z}^{T} \mathbf{Z}=\mathbf{I}, \mathbf{Q}^{T} \mathbf{Q}=\mathbf{I}\right\} .
$$

Both approaches proposed in this paper rely on a pre-processing step in which optimal transformations $\mathbf{Q}^{\star}\left(\boldsymbol{\mu}_{i}\right)$ and $\mathbf{Z}^{\star}\left(\boldsymbol{\mu}_{i}\right), i=1, \cdots, N_{\mathrm{DB}}$ are applied to the $N_{\text {DB }}$ ROMs stored in the database $\mathcal{D B}$.

The first approach, originally introduced in [17] and described in Section 3.2, is applicable whenever the underlying HDMs are defined on the same reference mesh. The position of the nodes of this reference mesh can be parameter-dependent, but its topology must be the same for all parameter values. This requirement is relaxed in Section 3.3 where a novel approach is introduced to enforce consistency in the case of arbitrary meshes. In that case, each HDM can have a different number of degrees of freedom (dofs).

A word of caution should be raised regarding the consistency and interpolation of reduced linear operators. There are cases for which consistency cannot be achieved with a right transformation. For example, consider the case of two configurations $\boldsymbol{\mu}_{1}$ and $\boldsymbol{\mu}_{2}$ associated with a common mesh and for which the right ROBs $\mathbf{V}\left(\boldsymbol{\mu}_{1}\right)$ and $\mathbf{V}\left(\boldsymbol{\mu}_{2}\right)$ are orthogonal to each other. In this case, the subspaces respectively defined by the ROBs are orthogonal as well and no transformation of the form $\left\{\mathbf{V}\left(\boldsymbol{\mu}_{i}\right) \mathbf{Q}\left(\boldsymbol{\mu}_{i}\right)\right\}_{i=1}^{2}$ can define a consistent set of reduced coordinates: the ROBs will remain orthogonal to each other. Such cases usually originate from a parameter variation that is too large or a discontinuous step in the computation of the ROB. Mode switching and truncation of a mode out of the ROB is an example. In this case, the truncated basis vector cannot be subsequently matched with its non-truncated counterpart. For this reason, the degree of consistency between two ROBs will be quantitatively defined in the case of common underlying meshes in Section 3.2, and a truncation procedure will be introduced to enforce consistency.

\subsection{Case of a common underlying mesh}

Consistency can be enforced in the case of a common underlying mesh by solving a series of Procrustes problems [17]. Specifically, given two local right reduced bases $\mathbf{V}_{i}=\mathbf{V}\left(\boldsymbol{\mu}_{i}\right)$ and $\mathbf{V}_{j}=\mathbf{V}\left(\boldsymbol{\mu}_{j}\right), \mathbf{V}_{j}$ can be written in terms of $\mathbf{V}_{i}$ as

$$
\mathbf{V}_{j}=\mathbf{V}_{i} \mathbf{R}_{i j}+\mathbf{T}_{i j}
$$

where $\mathbf{T}_{i j}$ is the component of $\mathbf{V}_{j}$ that is $\mathcal{M}$-orthogonal to $\mathbf{V}_{i}$ i.e. $\mathbf{T}_{i j}^{T} \mathcal{M} \mathbf{V}_{i}=\mathbf{0}$.

The subspace angles between the ROBs $\mathbf{V}_{i}$ and $\mathbf{V}_{j}$ define a measure of the maximum achievable consistency. They can be computed using the following three-step procedure:

1. Form $\mathbf{V}_{i}^{T} \mathcal{M} \mathbf{V}_{j}=\mathbf{R}_{i j}$.

2. Compute a singular value decomposition $\mathbf{R}_{i j}=\mathbf{X} \boldsymbol{\Sigma} \mathbf{Y}^{T}$ where $\mathbf{X}=\left[\mathbf{x}_{1}, \ldots, \mathbf{x}_{k}\right], \mathbf{Y}=\left[\mathbf{y}_{1}, \ldots, \mathbf{y}_{k}\right]$ and $\boldsymbol{\Sigma}=\operatorname{diag}\left(\sigma_{1}, \cdots, \sigma_{k}\right)$.

3. Compute the subspace angles as $\theta_{\ell}=\arccos \left(\sigma_{\ell}\right), \ell=1, \cdots, k$. The canonical vectors associated with each angle $\theta_{\ell}$ are $\left(\mathbf{V}_{i} \mathbf{x}_{\ell}, \mathbf{V}_{j} \mathbf{y}_{\ell}\right)$. Note that the angles are ordered increasingly as $0 \leq \theta_{1} \leq \cdots \leq \theta_{k} \leq \frac{\pi}{2}$.

A subspace angle $\theta_{\ell}$ that is equal to zero reflects perfect consistency between the associated vectors $\mathbf{V}_{i} \mathbf{x}_{\ell}$ and $\mathbf{V}_{j} \mathbf{y}_{\ell}$. In general, angles that are greater than a threshold value $\theta_{\max }=\frac{\pi}{4}$ may define cases for which consistency cannot be achieved, as in the mode switching example mentioned in the previous section. One option to address this issue is to truncate the directions associated with large angles. This option is discussed 
below. An alternative option is to refine the database until smaller subspace angles are achieved between neighboring ROMs so that they can be consistently interpolated.

For a given database $\mathcal{D B}$, optimal transformations $\left\{\mathbf{Q}\left(\boldsymbol{\mu}_{i}\right)\right\}_{i=1}^{N_{\mathrm{DB}}}$ can be computed by fixing one of the ROBs (say $\mathbf{Q}\left(\boldsymbol{\mu}_{i_{0}}\right)=\mathbf{I}_{k}$ ) and computing the transformations as the minimizers of the following Procrustes problems:

$$
\begin{aligned}
\mathbf{Q}\left(\boldsymbol{\mu}_{i}\right)=\underset{\mathbf{S} \in \mathbb{R}^{k \times k}}{\operatorname{argmin}}\left\|\mathbf{V}_{i} \mathbf{S}-\mathbf{V}_{i_{0}}\right\|_{\mathcal{M}}, \quad i=1, \cdots, N_{\mathrm{DB}}, \\
\text { s.t. } \mathbf{S}^{T} \mathbf{S}=\mathbf{I}_{k},
\end{aligned}
$$

where $\|\mathbf{N}\|_{\mathcal{M}}=\left\|\mathcal{M}^{\frac{1}{2}} \mathbf{N}\right\|_{F}$. The optimal transformation $\mathbf{Q}\left(\boldsymbol{\mu}_{i}\right)$ can be determined analytically from the SVD of $\mathbf{R}_{i i_{0}}$ as

$$
\mathbf{Q}\left(\boldsymbol{\mu}_{i}\right)=\mathbf{X Y}^{T}
$$

As stated above, truncation of the ROBs can be used to enforce consistency. For each ROB $\mathbf{V}_{i}, i=$ $1, \cdots, N_{\mathrm{DB}}$, the maximum index $\ell_{i}$ for which the subspace angles with $\mathbf{V}_{i_{0}}$ are smaller than $\theta_{\max }$ can be determined and all ROM can be truncated to the index

$$
L=\min _{i=1, \cdots, N_{\mathrm{DB}}} \ell_{i}
$$

Truncating the ROMs will result in a loss of accuracy of each ROM when compared to the underlying HDM. However, this truncation step will improve accuracy after interpolation of neighboring elements of the database as these will be more consistent.

Remark 1. An alternative approach to solving a sequence of Procrustes problems as explained above is to define a reference ROB with respect to which all other ROBs should be matched, and consider for this purpose solving a Generalized Procrustes Problem. In this case, an iterative procedure can be used to construct the reference ROB as an average of all ROBs. This alternative approach is not adopted here because it is more computationally intensive.

\subsection{Case of arbitrary underlying meshes}

In the case of arbitrary meshes, subspace angles cannot be defined as the underlying HDM spaces may be of different, parameter-dependent dimensions $N\left(\boldsymbol{\mu}_{i}\right)$. To address this issue, a new procedure is developed here to enforce consistency for this specific scenario. As in the previous case, it is still assumed that all the ROMs have a common reduced dimension. Also, as in the case of the Procrustes problem, one of the ROMs $\mathcal{R}_{i_{0}}$ is selected to define a reference configuration. Then, for each $\mathrm{ROM} \mathcal{R}_{i}, i=1, \cdots, N_{\mathrm{DB}}$ in the database, a transformed ROM $\mathcal{R}_{i}^{\star}$ is computed as the minimizer of a measure of a distance between the reference ROM $\mathcal{R}_{i_{0}}$ and the equivalence class $\mathcal{C}\left(\mathcal{R}_{i}\right)$ as follows

$$
\mathcal{R}_{i}^{\star}=\underset{\mathcal{R} \in \mathcal{C}\left(\mathcal{R}_{i}\right)}{\operatorname{argmin}} D_{i_{0}}\left(\mathcal{R}, \mathcal{R}_{i_{0}}\right) .
$$

The above minimization problem is schematically depicted in Fig. 1.

The following measure of the distance $D_{i_{0}}\left(\mathcal{R}, \mathcal{R}_{i_{0}}\right)$ is proposed:

1. For a first-order system, the following definition of a distance between $\mathcal{R}=\left(\mathbf{E}_{r}, \mathbf{A}_{r}, \mathbf{B}_{r}, \mathbf{G}_{r}, \mathbf{H}\right)$ and $\mathcal{R}^{\prime}=\left(\mathbf{E}_{r}^{\prime}, \mathbf{A}_{r}^{\prime}, \mathbf{B}_{r}^{\prime}, \mathbf{G}_{r}^{\prime}, \mathbf{H}^{\prime}\right)$ is proposed

$$
D_{i_{0}}\left(\mathcal{R}, \mathcal{R}^{\prime}\right)=\epsilon\left\|\mathbf{E}_{r}-\mathbf{E}_{r}^{\prime}\right\|_{F}^{2}+\alpha\left\|\mathbf{A}_{r}-\mathbf{A}_{r}^{\prime}\right\|_{F}^{2}+\beta\left\|\mathbf{B}_{r}-\mathbf{B}_{r}^{\prime}\right\|_{F}^{2}+\gamma\left\|\mathbf{G}_{r}-\mathbf{G}_{r}^{\prime}\right\|_{F}^{2}+\eta\left\|\mathbf{H}-\mathbf{H}^{\prime}\right\|_{F}^{2},
$$

where

$$
\epsilon=\frac{1}{\left\|\mathbf{E}_{r}^{0}\right\|_{F}^{2}}, \alpha=\frac{1}{\left\|\mathbf{A}_{r}^{0}\right\|_{F}^{2}}, \beta=\frac{1}{\left\|\mathbf{B}_{r}^{0}\right\|_{F}^{2}}, \gamma=\frac{1}{\left\|\mathbf{G}_{r}^{0}\right\|_{F}^{2}}, \eta=\frac{1}{\left\|\mathbf{H}^{0}\right\|_{F}^{2}}
$$

are normalization constants based on the linear reduced operators in $\mathcal{R}_{i_{0}}=\left(\mathbf{E}_{r}^{0}, \mathbf{A}_{r}^{0}, \mathbf{B}_{r}^{0}, \mathbf{G}_{r}^{0}, \mathbf{H}^{0}\right)$. 


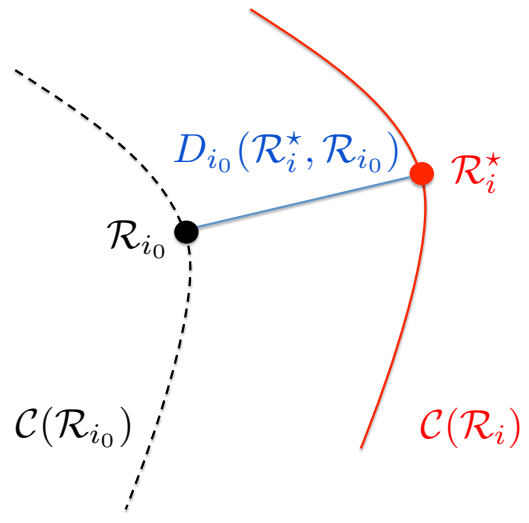

Figure 1 Case of different underlying meshes: distance minimization for enforcing consistency between ROMs

2. For a second-order system, the following definition of a distance between $\mathcal{R}=\left(\mathbf{M}_{r}, \mathbf{C}_{r}, \mathbf{K}_{r}, \mathbf{B}_{r}, \mathbf{G}_{r}, \mathbf{H}\right)$ and $\mathcal{R}^{\prime}=\left(\mathbf{M}_{r}^{\prime}, \mathbf{C}_{r}^{\prime}, \mathbf{K}_{r}^{\prime}, \mathbf{B}_{r}^{\prime}, \mathbf{G}_{r}^{\prime}, \mathbf{H}^{\prime}\right)$ is proposed

$D_{i_{0}}\left(\mathcal{R}, \mathcal{R}^{\prime}\right)=\mu\left\|\mathbf{M}_{r}-\mathbf{M}_{r}^{\prime}\right\|_{F}^{2}+\xi\left\|\mathbf{C}_{r}-\mathbf{C}_{r}^{\prime}\right\|_{F}^{2}+\kappa\left\|\mathbf{K}_{r}-\mathbf{K}_{r}^{\prime}\right\|_{F}^{2}+\beta\left\|\mathbf{B}_{r}-\mathbf{B}_{r}^{\prime}\right\|_{F}^{2}+\gamma\left\|\mathbf{G}_{r}-\mathbf{G}_{r}^{\prime}\right\|_{F}^{2}+\eta\left\|\mathbf{H}-\mathbf{H}^{\prime}\right\|_{F}^{2}$,

where

$$
\mu=\frac{1}{\left\|\mathbf{M}_{r}^{0}\right\|_{F}^{2}}, \quad \xi=\frac{1}{\left\|\mathbf{C}_{r}^{0}\right\|_{F}^{2}}, \quad \kappa=\frac{1}{\left\|\mathbf{K}_{r}^{0}\right\|_{F}^{2}}
$$

are normalization constants based on the linear reduced operators in $\mathcal{R}_{i_{0}}=\left(\mathbf{M}_{r}^{0}, \mathbf{C}_{r}^{0}, \mathbf{K}_{r}^{0}, \mathbf{B}_{r}^{0}, \mathbf{G}_{r}^{0}, \mathbf{H}^{0}\right)$.

In practice, since the class $\mathcal{C}\left(\mathcal{R}_{i}\right)$ is parameterized by two transformation matrices $\mathbf{Q}$ and $\mathbf{Z}$, the optimization problem (5) can be explicitly written for a first-order system in terms of $\mathcal{R}_{i}=\left(\mathbf{E}_{r i}, \mathbf{A}_{r i}, \mathbf{B}_{r i}, \mathbf{G}_{r i}, \mathbf{H}_{i}\right)$, $\mathcal{R}_{i_{0}}$ and $(\mathbf{Q}, \mathbf{Z})$ as

$$
\begin{aligned}
\min _{\mathbf{Q}, \mathbf{Z}} & \epsilon\left\|\mathbf{Z}^{T} \mathbf{E}_{r i} \mathbf{Q}-\mathbf{E}_{r}^{0}\right\|_{F}^{2}+\alpha\left\|\mathbf{Z}^{T} \mathbf{A}_{r} \mathbf{Q}-\mathbf{A}_{r}^{0}\right\|_{F}^{2}+\beta\left\|\mathbf{Z}^{T} \mathbf{B}_{r}-\mathbf{B}_{r}^{0}\right\|_{F}^{2}+\gamma\left\|\mathbf{G}_{r} \mathbf{Q}-\mathbf{G}_{r}^{0}\right\|_{F}^{2}+\eta\left\|\mathbf{H}-\mathbf{H}^{0}\right\|_{F}^{2} \\
\text { s.t. } & \mathbf{Q}^{T} \mathbf{Q}=\mathbf{I}_{k}, \quad \mathbf{Z}^{T} \mathbf{Z}=\mathbf{I}_{k} .
\end{aligned}
$$

A similar expression holds for second-order systems.

The rest of this section focuses only on first-order systems; however, its approach is equally applicable to second-order systems.

Remark 2. In the case of the Galerkin projection, $\mathbf{W}\left(\boldsymbol{\mu}_{i}\right)=\mathbf{V}\left(\boldsymbol{\mu}_{i}\right)$ and $\mathbf{Q}=\mathbf{Z}$. In this case, (5) simplifies to

$$
\begin{aligned}
& \min _{\mathbf{Q}} \epsilon\left\|\mathbf{Q}^{T} \mathbf{E}_{r i} \mathbf{Q}-\mathbf{E}_{r}^{0}\right\|_{F}^{2}+\alpha\left\|\mathbf{Q}^{T} \mathbf{A}_{r} \mathbf{Q}-\mathbf{A}_{r}^{0}\right\|_{F}^{2}+\beta\left\|\mathbf{Q}^{T} \mathbf{B}_{r}-\mathbf{B}_{r}^{0}\right\|_{F}^{2}+\gamma\left\|\mathbf{G}_{r} \mathbf{Q}-\mathbf{G}_{r}^{0}\right\|_{F}^{2}+\eta\left\|\mathbf{H}-\mathbf{H}^{0}\right\|_{F}^{2} \\
& \text { s.t. } \mathbf{Q}^{T} \mathbf{Q}=\mathbf{I}_{k} .
\end{aligned}
$$

Problem (6) is equivalent to the maximization problem [14]

$$
\begin{aligned}
& \max _{\mathbf{Q}, \mathbf{Z}}\left\langle\epsilon \mathbf{Z}^{T} \mathbf{E}_{r i} \mathbf{Q}, \mathbf{E}_{r}^{0}\right\rangle+\left\langle\alpha \mathbf{Z}^{T} \mathbf{A}_{r i} \mathbf{Q}, \mathbf{A}_{r}^{0}\right\rangle+\left\langle\beta \mathbf{B}_{r}\left(\mathbf{B}_{r}^{0}\right)^{T}, \mathbf{Z}\right\rangle+\left\langle\gamma\left(\mathbf{G}_{r}^{0}\right)^{T} \mathbf{G} r, \mathbf{Q}\right\rangle \\
& \text { s.t. } \mathbf{Q}^{T} \mathbf{Q}=\mathbf{I}_{k}, \quad \mathbf{Z}^{T} \mathbf{Z}=\mathbf{I}_{k},
\end{aligned}
$$

where

$$
\langle\mathbf{M}, \mathbf{N}\rangle=\operatorname{tr}\left(\mathbf{M}^{T} \mathbf{N}\right), \quad \mathbf{M}, \mathbf{N} \in \mathbb{R}^{m \times n}
$$


The problem of maximizing the first term in Eq. (8) was studied in $[21,22]$ in the case where $\mathbf{Q}=\mathbf{Z}$ (Galerkin projection). This first term defines a correlation criterion between the matrices $\mathbf{E}_{r i}$ and $\mathbf{E}_{r}^{0}$. A solution to this problem was developed in [21]. It consists of constructing an iterative algorithm whose fixed points are the stationary points of the maximization problem. This approach is extended below to the optimization problem of interest - that is, that defined for enforcing consistency between ROM operators. Both Galerkin and Petrov-Galerkin projections are considered.

- Case of the Galerkin projection:

In this case, the functional in (8) is

$$
\mathcal{J}_{G}(\mathbf{Q})=\left\langle\epsilon \mathbf{Q}^{T} \mathbf{E}_{r i} \mathbf{Q}, \mathbf{E}_{r}^{0}\right\rangle+\left\langle\alpha \mathbf{Q}^{T} \mathbf{A}_{r i} \mathbf{Q}, \mathbf{A}_{r}^{0}\right\rangle+\left\langle\beta \mathbf{B}_{r}\left(\mathbf{B}_{r}^{0}\right)^{T}, \mathbf{Q}\right\rangle+\left\langle\gamma\left(\mathbf{G}_{r}^{0}\right)^{T} \mathbf{G}_{r}, \mathbf{Q}\right\rangle .
$$

Adapting the algorithm developed in [21] to this case leads to an iterative algorithm that is based on the following affine map

$$
\mathbf{M}_{s, \mathrm{G}}(\mathbf{Q})=\epsilon\left(\mathbf{E}_{r i} \mathbf{Q}\left(\mathbf{E}_{r}^{0}\right)^{T}+\mathbf{E}_{r i}^{T} \mathbf{Q} \mathbf{E}_{r}^{0}\right)+\alpha\left(\mathbf{A}_{r i} \mathbf{Q}\left(\mathbf{A}_{r}^{0}\right)^{T}+\mathbf{A}_{r i}^{T} \mathbf{Q} \mathbf{A}_{r}^{0}\right)+s \mathbf{Q}+\beta \mathbf{B}_{r i}\left(\mathbf{B}_{r}^{0}\right)^{T}+\gamma \mathbf{G}_{r i}^{T} \mathbf{G}_{r}^{0},
$$

where $s$ is a fixed real parameter chosen such that $s>s_{\min , \mathrm{G}}$ with

$$
s_{\min , \mathrm{G}}=2 \epsilon\left\|\mathbf{E}_{r i}\right\|_{2}\left\|\mathbf{E}_{r}^{0}\right\|_{2}+2 \alpha\left\|\mathbf{A}_{r i}\right\|_{2}\left\|\mathbf{A}_{r}^{0}\right\|_{2}+\left\|\beta \mathbf{B}_{r i}\left(\mathbf{B}_{r}^{0}\right)^{T}+\gamma \mathbf{G}_{r i}^{T} \mathbf{G}_{r}^{0}\right\|_{2} .
$$

Defining the parameter $s$ is necessary to ensure that the fixed points of the proposed iterative algorithm are exactly the stationary points of the maximization problem of interest (see Theorem 1 ). Then, the proposed procedure proceeds by iteratively solving the maximization problem

$$
\mathbf{Q}_{j+1}=\arg \max _{\mathbf{Q}^{T} \mathbf{Q}=\mathbf{I}_{k}}\left\langle\mathbf{Q}, \mathbf{M}_{s, \mathrm{G}}\left(\mathbf{Q}_{j}\right)\right\rangle, \quad j=0, \cdots .
$$

The solution of this problem is established in the following lemma, leading to the iterative procedure presented in Algorithm 1.

Lemma. Let the singular value decomposition of $\mathbf{M}_{s, \mathrm{G}}\left(\mathbf{Q}_{j}\right)$ be defined as

$$
\mathbf{M}_{s, \mathrm{G}}\left(\mathbf{Q}_{j}\right)=\mathbf{U} \boldsymbol{\Sigma} \mathbf{V}^{T}
$$

Then

$$
\max _{\mathbf{Q}^{T} \mathbf{Q}=\mathbf{I}_{k}}\left\langle\mathbf{Q}, \mathbf{M}_{s}\left(\mathbf{Q}_{j}\right)\right\rangle=\sum_{\ell=1}^{k} \sigma_{\ell}\left(\mathbf{M}_{s, \mathrm{G}}\left(\mathbf{Q}_{j}\right)\right),
$$

where $\left\{\sigma_{\ell}\left(\mathbf{M}_{s, \mathrm{G}}\left(\mathbf{Q}_{j}\right)\right)\right\}_{\ell=1}^{k}$ is the set of singular values of $\mathbf{M}_{s, \mathrm{G}}\left(\mathbf{Q}_{j}\right)$. The solution of the above maximization problem is unique and equal to $\mathbf{U V}^{T}$.

A proof of the above lemma can be found in [21], albeit in a more general setting.

In order to show that the fixed points of the above iterative algorithm are the stationary points of $\mathcal{J}_{G}$, one needs to characterize these stationary points. This is done in the following theorem.

Theorem 1. The stationary points $\overline{\mathbf{Q}}$ of $\mathcal{J}_{G}$ are orthogonal matrices that satisfy the identity

$$
\overline{\mathbf{Q}} \mathbf{S}=\epsilon\left(\mathbf{E}_{r i} \overline{\mathbf{Q}}\left(\mathbf{E}_{r}^{0}\right)^{T}+\mathbf{E}_{r i}^{T} \overline{\mathbf{Q}} \mathbf{E}_{r}^{0}\right)+\alpha\left(\mathbf{A}_{r i} \overline{\mathbf{Q}}\left(\mathbf{A}_{r}^{0}\right)^{T}+\mathbf{A}_{r i}^{T} \overline{\mathbf{Q}} \mathbf{A}_{r}^{0}\right)+\beta \mathbf{B}_{r i}\left(\mathbf{B}_{r}^{0}\right)^{T}+\gamma \mathbf{G}_{r i}^{T} \mathbf{G}_{r}^{0}
$$

where $\mathbf{S}$ is a symmetric matrix.

A proof of the above theorem is offered in Appendix 1.

Theorem 2. The set of fixed points of Algorithm 1 is exactly the set of stationary points of $\mathcal{J}_{G}$.

A proof of the above theorem is presented in Appendix 2. 
Algorithm 1 Fixed-point iterative procedure for the case of the Galerkin projection.

Inputs: normalization constants $\epsilon, \alpha, \beta, \gamma$, matrices $\mathbf{E}_{r i}, \mathbf{A}_{r i}, \mathbf{B}_{r i}, \mathbf{G}_{r i}$, matrices $\mathbf{E}_{r}^{0}, \mathbf{A}_{r}^{0}, \mathbf{B}_{r}^{0}, \mathbf{G}_{r}^{0}$. Output: Orthogonal matrix $\mathbf{Q}_{j+1}$ for terminating $j$.

1: Compute $s>2 \epsilon\left\|\mathbf{E}_{r i}\right\|_{2}\left\|\mathbf{E}_{r}^{0}\right\|_{2}+2 \alpha\left\|\mathbf{A}_{r i}\right\|_{2}\left\|\mathbf{A}_{r}^{0}\right\|_{2}+\left\|\beta \mathbf{B}_{r i}\left(\mathbf{B}_{r}^{0}\right)^{T}+\gamma \mathbf{G}_{r i}^{T} \mathbf{G}_{r}^{0}\right\|_{2}$.

2: Compute $\mathbf{F}=\beta \mathbf{B}_{r i}\left(\mathbf{B}_{r}^{0}\right)^{T}+\gamma \mathbf{G}_{r i}^{T} \mathbf{G}_{r}^{0}$.

3: Choose an orthogonal initial matrix $\mathbf{Q}_{0} \in \mathbb{R}^{k \times k}$.

4: for $j=0, \cdots$ do

5: $\quad$ Compute the map

$$
\mathbf{M}_{s, \mathrm{G}}\left(\mathbf{Q}_{j}\right)=\epsilon\left(\mathbf{E}_{r i} \mathbf{Q}_{j}\left(\mathbf{E}_{r}^{0}\right)^{T}+\mathbf{E}_{r i}^{T} \mathbf{Q}_{j} \mathbf{E}_{r}^{0}\right)+\alpha\left(\mathbf{A}_{r i} \mathbf{Q}_{j}\left(\mathbf{A}_{r}^{0}\right)^{T}+\mathbf{A}_{r i}^{T} \mathbf{Q}_{j} \mathbf{A}_{r}^{0}\right)+s \mathbf{Q}_{j}+\mathbf{F}
$$

6: $\quad$ Compute its SVD

$$
\mathbf{U}_{j+1} \boldsymbol{\Sigma}_{j+1} \mathbf{V}_{j+1}^{T}=\mathbf{M}_{s, \mathrm{G}}\left(\mathbf{Q}_{j}\right)
$$

7: $\quad \mathbf{Q}_{j+1}=\mathbf{U}_{j+1} \mathbf{V}_{j+1}^{T}$.

8: end for

- Case of the Petrov-Galerkin projection:

Defining the functional

$$
\mathcal{J}_{P G}(\mathbf{Q}, \mathbf{Z})=\left\langle\epsilon \mathbf{Z}^{T} \mathbf{E}_{r i} \mathbf{Q}, \mathbf{E}_{r}^{0}\right\rangle+\left\langle\alpha \mathbf{Z}^{T} \mathbf{A}_{r i} \mathbf{Q}, \mathbf{A}_{r}^{0}\right\rangle+\left\langle\beta \mathbf{B}_{r}\left(\mathbf{B}_{r}^{0}\right)^{T}, \mathbf{Z}\right\rangle+\left\langle\gamma\left(\mathbf{G}_{r}^{0}\right)^{T} \mathbf{G}_{r}, \mathbf{Q}\right\rangle,
$$

the iterative algorithm is now based on the block-affine map defined as

$$
\begin{aligned}
\mathbf{M}_{s, \mathrm{PG}}(\mathbf{Q}, \mathbf{Z})= & {\left[\begin{array}{cc}
\mathbf{M}_{s, \mathrm{PG}}^{Q}(\mathbf{Q}, \mathbf{Z}) & \mathbf{0} \\
\mathbf{0} & \mathbf{M}_{s, \mathrm{PG}}^{Z}(\mathbf{Q}, \mathbf{Z})
\end{array}\right] } \\
= & \epsilon\left[\begin{array}{cc}
\mathbf{0} & \mathbf{E}_{r i} \\
\mathbf{E}_{r i}^{T} & \mathbf{0}
\end{array}\right]\left[\begin{array}{cc}
\mathbf{Q} & \mathbf{0} \\
\mathbf{0} & \mathbf{Z}
\end{array}\right]\left[\begin{array}{cc}
\mathbf{0} & \left(\mathbf{E}_{r}^{0}\right)^{T} \\
\left(\mathbf{E}_{r}^{0}\right)^{T} & \mathbf{0}
\end{array}\right] \\
& +\alpha\left[\begin{array}{cc}
\mathbf{0} & \mathbf{A}_{r i} \\
\mathbf{A}_{r i}^{T} & \mathbf{0}
\end{array}\right]\left[\begin{array}{cc}
\mathbf{Q} & \mathbf{0} \\
\mathbf{0} & \mathbf{Z}
\end{array}\right]\left[\begin{array}{cc}
\mathbf{0} & \left(\mathbf{A}_{r}^{0}\right)^{T} \\
\left(\mathbf{A}_{r}^{0}\right)^{T} & \mathbf{0}
\end{array}\right] \\
& +s\left[\begin{array}{cc}
\mathbf{Q} & \mathbf{0} \\
\mathbf{0} & \mathbf{Z}
\end{array}\right]+\left[\begin{array}{cc}
\beta \mathbf{B}_{r i}\left(\mathbf{B}_{r}^{0}\right)^{T} & \mathbf{0} \\
\mathbf{0} & \gamma \mathbf{G}_{r i}^{T} \mathbf{G}_{r}^{0}
\end{array}\right],
\end{aligned}
$$

where $s$ is chosen such that $s>s_{\text {min,PG }}$ with

$$
s_{\min , \mathrm{PG}}=\epsilon\left\|\mathbf{E}_{r i}\right\|_{2}\left\|\mathbf{E}_{r}^{0}\right\|_{2}+\alpha\left\|\mathbf{A}_{r i}\right\|_{2}\left\|\mathbf{A}_{r}^{0}\right\|_{2}+\max \left(\beta\left\|\mathbf{B}_{r i}\left(\mathbf{B}_{r}^{0}\right)^{T}\right\|_{2}, \gamma\left\|\mathbf{G}_{r i}^{T} \mathbf{G}_{r}^{0}\right\|_{2}\right) .
$$

As in the case of the Galerkin projection, a fixed-point iterative procedure is proposed in Algorithm 2.

The following theorems, whose proofs follow closely those of Theorems 1 and 2, establish the fact that the fixed-point iterative procedure given in Algorithm 2 can be used to find the stationary points of $\mathcal{J}_{P G}$.

Theorem 3 The stationary points $(\overline{\mathbf{Q}}, \overline{\mathbf{Z}})$ of $\mathcal{J}_{P G}$ are orthogonal matrices satisfying the identities

$$
\begin{aligned}
\overline{\mathbf{Q}} \mathbf{S}_{Q} & =\epsilon \mathbf{E}_{r i} \overline{\mathbf{Z}}\left(\mathbf{E}_{r}^{0}\right)^{T}+\alpha \mathbf{A}_{r i} \overline{\mathbf{Z}}\left(\mathbf{A}_{r}^{0}\right)^{T}+\gamma \mathbf{G}_{r i}^{T} \mathbf{G}_{r}^{0}, \\
\overline{\mathbf{Z}} \mathbf{S}_{Z} & =\epsilon \mathbf{E}_{r i}^{T} \overline{\mathbf{Q}}\left(\mathbf{E}_{r}^{0}\right)^{T}+\alpha \mathbf{A}_{r i}^{T} \overline{\mathbf{Q}}\left(\mathbf{A}_{r}^{0}\right)^{T}+\beta \mathbf{B}_{r i}\left(\mathbf{B}_{r}^{0}\right)^{T},
\end{aligned}
$$




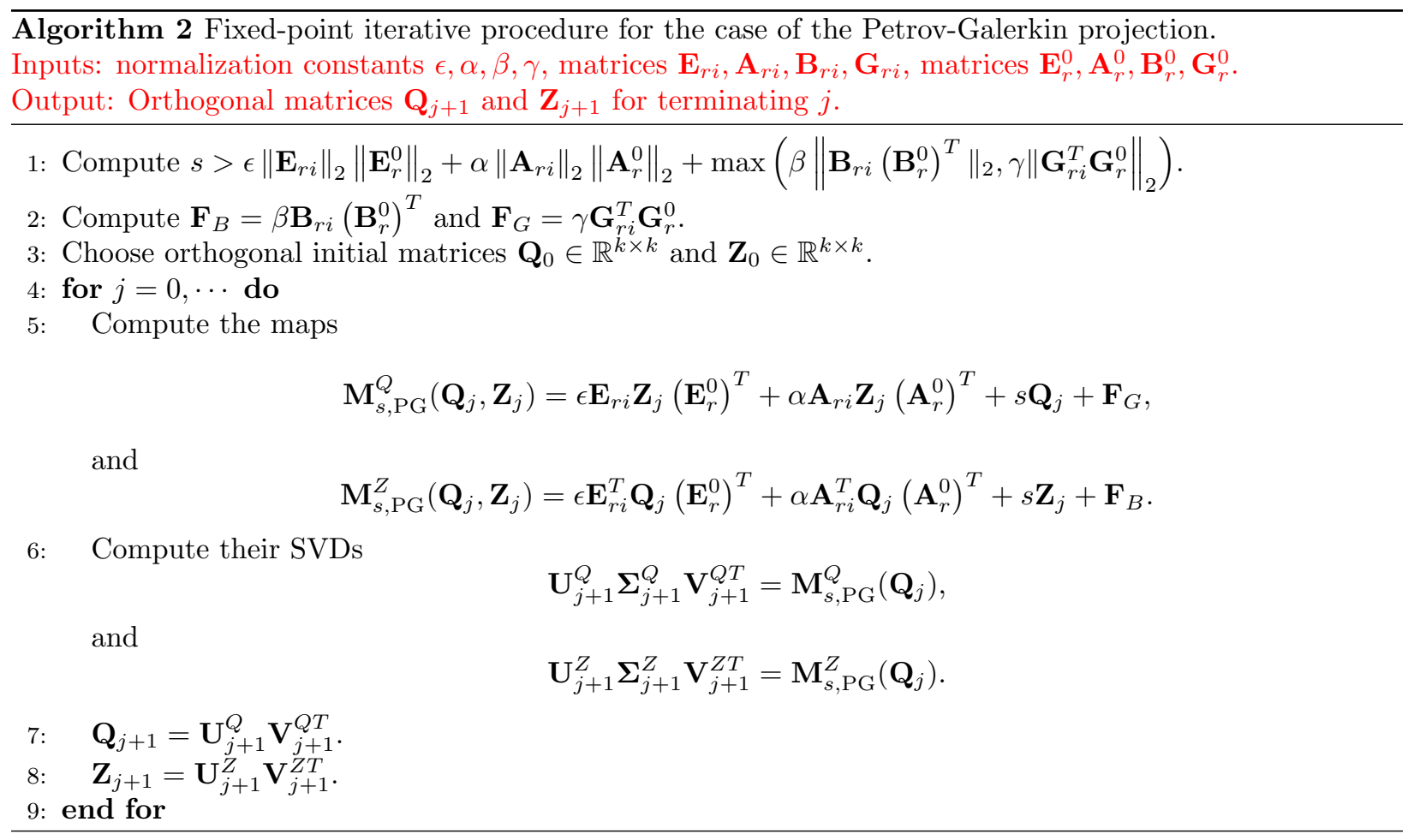

where $\mathbf{S}_{Q}$ and $\mathbf{S}_{Z}$ are symmetric matrices.

Theorem 4. The set of fixed points of the recursive Algorithm 2 is exactly the set of stationary points of $\mathcal{J}_{P G}$.

Remark 3. In the case of arbitrary meshes, assessing consistency is a more difficult task as two conflicting factors intervene in the distance measure $D_{i_{0}}$ : (1) the inconsistency arising from a choice of two distinct sets of coordinates, and (2) the inherent variation of the ROM operators due to parameter changes.

\subsection{Consistent set of reduced-order models}

After the computation of the optimal transformation operators $\left\{\left(\overline{\mathbf{Z}}\left(\boldsymbol{\mu}_{i}\right), \overline{\mathbf{Q}}\left(\boldsymbol{\mu}_{i}\right)\right)\right\}_{i=1}^{N_{\mu}}$ is performed, the linear reduced operators in the database $\mathcal{D B}$ are transformed accordingly as follows

$$
\begin{aligned}
\mathcal{D B} & \left.=\left\{\boldsymbol{\mu}_{i} ; \mathbf{E}_{r}^{\star}\left(\boldsymbol{\mu}_{i}\right), \mathbf{A}_{r}^{\star}\left(\boldsymbol{\mu}_{i}\right), \mathbf{B}_{r}^{\star}\left(\boldsymbol{\mu}_{i}\right), \mathbf{G}_{r}^{\star}\left(\boldsymbol{\mu}_{i}\right), \mathbf{H}_{r}^{\star}\left(\boldsymbol{\mu}_{i}\right)\right)\right\}_{i=1}^{N_{\mu}} \\
& =\left\{\boldsymbol{\mu}_{i} ;\left(\overline{\mathbf{Z}}\left(\boldsymbol{\mu}_{i}\right)^{T} \mathbf{E}_{r}\left(\boldsymbol{\mu}_{i}\right) \overline{\mathbf{Q}}\left(\boldsymbol{\mu}_{i}\right), \overline{\mathbf{Z}}\left(\boldsymbol{\mu}_{i}\right)^{T} \mathbf{A}_{r}\left(\boldsymbol{\mu}_{i}\right) \overline{\mathbf{Q}}\left(\boldsymbol{\mu}_{i}\right), \overline{\mathbf{Z}}\left(\boldsymbol{\mu}_{i}\right)^{T} \mathbf{B}_{r}\left(\boldsymbol{\mu}_{i}\right), \mathbf{G}_{r}^{\star}\left(\boldsymbol{\mu}_{i}\right) \overline{\mathbf{Q}}\left(\boldsymbol{\mu}_{i}\right), \mathbf{H}_{r}\left(\boldsymbol{\mu}_{i}\right)\right)\right\}_{i=1}^{N_{\mu}} .
\end{aligned}
$$

Similar expressions hold for the case of second-order systems.

\section{Construction and manipulation of a database of ROMs}

\subsection{Sampling}

The selection of sample parameter points $\left\{\boldsymbol{\mu}_{i}\right\}_{i=1}^{N_{D B}}$ is an important step that influences the accuracy of the resulting interpolation procedure. A poor choice of sample points will typically result in large interpolation errors, at least in some regions of the parameter domain $\mathcal{D}$. A priori sampling techniques such as factorial and latin hypercube sampling can be used to provide a uniform coverage of $\mathcal{D}$. Alternatively, greedy techniques that iteratively sample the regions of the parameter space associated with the largest ROM error can provide a selection of sample parameter points that is more suited for the application of interest $[10,23,24]$. In this paper, an à priori sampling technique is used for the application described in Section 5.2, and a greedy sampling approach is used for the parametric inverse problem discussed in Section 5.1. 


\subsection{Storage and exploitation}

In practice, the linear reduced operators are stored - after congruence transformations are performed for enforcing consistency - in one database $\mathcal{D B}$ or multiple sub-databases $\{\mathcal{D B}\}_{s=1}^{N_{s}}$ of the form

$$
\mathcal{D B}=\bigcup_{s=1}^{N_{S}} \mathcal{D} \mathcal{B}_{s}=\bigcup_{s=1}^{N_{S}}\left\{\boldsymbol{\mu}_{i, s} ;\left(\mathbf{E}_{r}^{\star}\left(\boldsymbol{\mu}_{i, s}\right), \mathbf{A}_{r}^{\star}\left(\boldsymbol{\mu}_{i, s}\right), \mathbf{B}_{r}^{\star}\left(\boldsymbol{\mu}_{i, s}\right), \mathbf{G}_{r}^{\star}\left(\boldsymbol{\mu}_{i, s}\right), \mathbf{H}^{\star}\left(\boldsymbol{\mu}_{i, s}\right)\right)\right\}_{i=1}^{N_{D B, s}}
$$

for first-order systems, and

$$
\mathcal{D B}=\bigcup_{s=1}^{N_{S}} \mathcal{D} \mathcal{B}_{s}=\bigcup_{s=1}^{N_{S}}\left\{\boldsymbol{\mu}_{i, s} ;\left(\mathbf{M}_{r}^{\star}\left(\boldsymbol{\mu}_{i, s}\right), \mathbf{C}_{r}^{\star}\left(\boldsymbol{\mu}_{i, s}\right), \mathbf{K}_{r}^{\star}\left(\boldsymbol{\mu}_{i, s}\right), \mathbf{B}_{r}^{\star}\left(\boldsymbol{\mu}_{i, s}\right), \mathbf{G}_{r}^{\star}\left(\boldsymbol{\mu}_{i, s}\right), \mathbf{H}^{\star}\left(\boldsymbol{\mu}_{i, s}\right)\right)\right\}_{i=1}^{N_{D B, s}}
$$

for second-order ones. In general, storing a ROM database is inexpensive because a ROM is by definition a low-dimensional operator. In practice, a database with $N_{D B}=\sum_{s=1}^{S} N_{D B, s}$ reduced linear operators contains $N_{D B}\left(N_{\boldsymbol{\mu}}+2 k^{2}+k\left(N_{i}+N_{o}\right)+N_{i} N_{o}\right)$ matrix entries for first-order systems of the form (1), and $N_{D B}\left(N_{\boldsymbol{\mu}}+3 k^{2}+k\left(N_{i}+N_{o}\right)+N_{i} N_{o}\right)$ entries for second-order systems of the form (3).

Effectively, the parameter domain $\mathcal{D}$ is subdivided in $N_{S}$ subdomains $\left\{\mathcal{D}_{s}\right\}_{s=1}^{N_{S}}$ such that

$$
\mathcal{D}=\bigcup_{s=1}^{N_{S}} \mathcal{D}_{s}
$$

and each subdomain $\mathcal{D}_{s}$ is associated with the sub-database $\mathcal{D} \mathcal{B}_{s}$. Then, during the online phase of a linear ROM computation, for each queried value $\widehat{\boldsymbol{\mu}} \in \mathcal{D}$, the sub-database $\mathcal{D} \mathcal{B}_{s_{0}}$ containing this parameter vector is identified in real-time, and reduced-operators are computed at $\widehat{\boldsymbol{\mu}}$ - also in real-time - by interpolating an appropriate subset of the ROMs stored in $\mathcal{D B}_{s_{0}}$.

\subsection{Interpolation algorithms}

\subsubsection{Interpolation on the tangent space to a matrix manifold}

As already stated in Section 2, the interpolation of linear reduced operators stored in a database of linear ROMs $\mathcal{D B}$ should preserve, as applicable, some of the important algebraic properties enjoyed by these operators such as symmetry, positivity, orthogonality, or non-singularity. One approach for achieving this objective was first presented in [12], in the context of subspaces. This approach is based on interpolation on the tangent space to the manifold representing the mathematical property of interest. The "overall" interpolation procedure associated with this approach was successfully applied in $[15,17]$ to various linear, reduced operators with different mathematical properties. This procedure is organized in four steps as follows:

1. Step 1: Identify manifolds to which a reduced operator (matrix) belongs to, and select one of them.

2. Step 2: Map (logarithmic map) a subset of the database entries to the tangent space to this chosen manifold at a chosen reference point of the database.

3. Step 3: Interpolate the mapped entities in the tangent space at the target parameter point $\widehat{\boldsymbol{\mu}}$.

4. Step 4: Map (exponential map) the interpolated entity back to the chosen manifold to obtain a linear, reduced operator at the target parameter point $\widehat{\boldsymbol{\mu}}$.

Further details about the interpolation algorithms and specific mapping formulae for well-known matrix manifolds can be found in [17].

When multiple (and possibly nested) matrix manifolds are identified for characterizing a matrix of interest, the heuristic approach presented in [15] can be used to select the matrix manifold to adopt for the interpolation procedure outlined above. For example, this heuristic approach can be used to determine, for 
a queried parameter point, whether to interpolate a set of non-singular reduced matrices on the manifold of non-singular matrices or on that of square matrices of a given size. An illustration of this approach is discussed in Section 5.2 of this paper.

Remark 4. The following remarks concerning the overall interpolation procedure outlined above are noteworthy:

- In Step 3, there is no restriction on the interpolation algorithm to be applied in the tangent space to the chosen matrix manifold, as long as this algorithm delivers an interpolated entity that also lies on the tangent space [17].

- In [25], it was shown that the pchip (shape-preserving piecewise-cubic interpolation) algorithm in Matlab violates the aforementioned interpolation algorithm (Step 3) property.

- When the parameter space $\mathcal{D}$ is sampled on a lattice of parameter points, multivariate polynomial interpolations satisfy the aforementioned interpolation algorithm property and therefore can be considered for performing Step 3 of the overall interpolation procedure.

- When parameter sampling is not performed on a lattice of points - for example, because the dimension $N_{\mu}$ of $\mathcal{D}$ is rather large - interpolation algorithms based on radial basis functions or Kriging can be used instead [12] as they satisfy the aforementioned interpolation algorithm property [25].

\subsubsection{Special case: interpolation of SPD matrices}

For symmetric positive definite (SPD) matrices of size $k$ and linear interpolation, an alternative approach to interpolating such matrices on the tangent space to the manifold of SPD matrices of dimension $k, \operatorname{SPD}(k)$, is to simply interpolate the entries of the Cholesky factors of these matrices. This simple approach is described in Algorithm 3 and illustrated in Section 5.1. It has also the advantage of not requiring the selection of a reference point in the database. It preserves the SPD property as long as the entries of the interpolated diagonal of the Cholesky decomposition are all non-zero, which is always the case for linear interpolation.

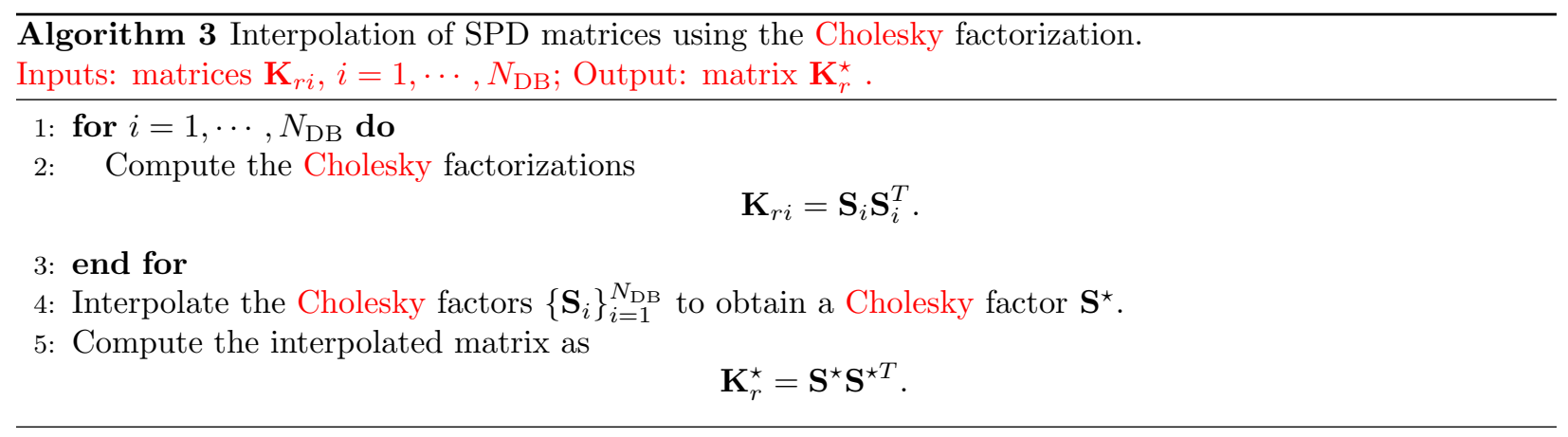

\section{Applications and performance assessment}

The proposed comprehensive approach for real-time computations using a database of parametric, linear, projection-based reduced-order models (ROMs) is illustrated here with two different applications: a parametric inverse acoustic scattering problem associated with a mockup submarine, and a parametric flutter prediction problem associated with a wing-tank system.

\subsection{Acoustic scattering}

\subsubsection{Problem formulation}

The parametric inverse acoustic scattering problem considered here consists in determining the shape of an obstacle - or a part of this shape - from the knowledge of a set of scattered far-field patterns 
"measured" at different frequencies (or equivalently, wavenumbers), assuming certain characteristics of its surface. It is well-known [26] that such an inverse problem is nonlinear and usually ill-posed, making its numerical solution challenging. Specifically, the shape of a given obstacle is assumed to be a known function of a set of parameters represented by the parameter vector $\boldsymbol{\mu} \in \mathbb{R}^{N_{\mu}}$, and the problem is defined as that of identifying the values of these parameters from the measured far-field patterns. The class of such problems is a subset of a more general inverse acoustic problem known as the inverse obstacle problem.

In order to describe the identification problem considered here more precisely, the direct acoustic scattering problem is first recalled.

Given an infinite homogeneous fluid medium $\Omega_{e} \subset \mathbb{R}^{d}$ and an impenetrable obstacle with a boundary $\Sigma$ embedded in this medium, the scattering of time-harmonic acoustic waves by this obstacle can be formulated as the following exterior boundary value problem in the acoustic fluid pressure $w$

$$
\begin{aligned}
\Delta w+\kappa^{2} w & =0 \quad \text { in } \quad \Omega_{e} \\
\left(a+b \frac{\partial}{\partial \boldsymbol{\nu}}\right)\left(w+w^{i n c}\right) & =0 \quad \text { on } \quad \Sigma, \\
\lim _{r \rightarrow \infty} r^{\frac{d-1}{2}}\left(\frac{\partial w}{\partial r}-j \kappa w\right) & =0
\end{aligned}
$$

where $w^{i n c}$ is an incident plane wave of the form

$$
w^{i n c}=e^{j \kappa \mathbf{d} \cdot \mathbf{x}}
$$

the unit vector $\mathbf{d}$ specifies the direction of incidence, and either $a \neq 0$ or $b \neq 0$. Sound-hard, sound-soft, or impedance boundary conditions can all be represented by the second of Eqs. (13). Here, the sound-hard case is considered and $a$ and $b$ are set to $a=0$ and $b=1$. The third of Eqs. (13) is the Sommerfeld radiation condition. From a physical viewpoint, it ensures that all waves are outgoing. From a mathematical one, it guarantees that the direct scattering problem is well-posed for any wavenumber $\kappa=\omega / c$, where $\omega$ denotes the angular frequency of the harmonic oscillations and $c$ denotes the speed of sound in the fluid medium.

In this example, the direct scattering problem (13) is discretized by a finite element method. To this effect, the infinite domain is first truncated, and a perfectly matched layer (PML) [27] is used near the exterior boundary to simulate the effect of the Sommerfeld condition. This transforms the boundary value problem (13) into the linear algebraic problem

$$
\left(\mathbf{K}(\boldsymbol{\mu})-\kappa^{2} \mathbf{M}(\boldsymbol{\mu})\right) \mathbf{w}(\kappa, \boldsymbol{\mu})=\mathbf{f}(\kappa, \boldsymbol{\mu})
$$

where $\mathbf{w} \in \mathbb{C}^{N}$ denotes the vector pressure dofs, $\mathbf{K}$ is a stiffness-type matrix that arises from the finite element discretization of the Laplace operator, $\mathbf{M}$ is a mass-type matrix, and $\mathbf{f}$ arises from the discretization of the sound-hard boundary condition.

For an interior variant of the above problem, $\mathbf{M}$ is real, symmetric positive-definite, and $\mathbf{K}$ is real, symmetric non-negative. For an exterior boundary value problem such as that considered here, the matrices $\mathbf{K}$ and $\mathbf{M}$ are complex and non-Hermitian.

The far-field pattern characterizes the asymptotic behavior of the acoustic scattered field far away from the obstacle. In two dimensions, it admits the following integral representation [26]

$$
w_{\infty}(\hat{\mathbf{x}})=\frac{e^{j \frac{\pi}{4}}}{(8 \pi \kappa)^{\frac{1}{2}}} \int_{\Gamma}\left(\frac{\partial w}{\partial \boldsymbol{\nu}}(\mathbf{y})+j \kappa \hat{\mathbf{x}} \cdot \boldsymbol{\nu} w(\mathbf{y})\right) e^{-j \kappa \hat{\mathbf{x}} \cdot \mathbf{y}} d \sigma_{y} ; \quad \hat{\mathbf{x}} \in S^{1},
$$

where $S^{1}$ denotes the unit circle. After computing the finite-element solution, the integral (15) is typically evaluated at $N_{o}$ locations $\hat{x}_{1}, \cdots, \hat{x}_{N_{o}}$ on the circle $S^{1}$ by integrating the integrand over a suitable curve $\Gamma$ in the computational domain (often the boundary $\Sigma$ ). The obtained results can be represented as the action of a matrix on the solution vector

$$
\mathbf{y}(\kappa, \boldsymbol{\mu})=\mathbf{G}(\kappa, \boldsymbol{\mu}) \mathbf{w}(\kappa, \boldsymbol{\mu}), \quad \mathbf{G} \in \mathbb{C}^{N_{o} \times N} .
$$


In practice however, the QoI is often defined as the related logarithmic scale quantity

$$
\mathcal{S}(\hat{\mathbf{x}})=10 \log _{10}\left(2 \pi\left|w_{\infty}(\hat{\mathbf{x}})\right|^{2}\right) .
$$

This QoI can be computed for each entry of the output vector $\mathbf{y}(\kappa, \boldsymbol{\mu})$ defined above as

$$
\mathbf{s}(\kappa, \boldsymbol{\mu})=\mathbf{s}(\mathbf{y}(\kappa, \boldsymbol{\mu}))
$$

More specifically, the parametric inverse problem considered here consists of identifying the parameter vector $\boldsymbol{\mu}=\left(L^{s}, t^{s}\right) \in \mathcal{D} \subset \mathbb{R}^{2}$ of a two-dimensional mockup submarine, where $L^{s}$ denotes its length and $t^{s}$ the position of its tower $t^{s}$ (Figure 2 (right)), from far-field data given for $N_{\kappa}$ different wavenumbers $\left\{\mathbf{s}_{m}\left(\kappa_{i}\right)\right\}_{i=1}^{N_{\kappa}}$. This problem can be formulated as a PDE (partial differential equation)-constrained minimization problem. After discretization, it can be written as

$$
\begin{aligned}
\min _{\boldsymbol{\mu} \in \mathcal{D}} \sum_{i=1}^{N_{\kappa}} \alpha_{i}\left\|\mathbf{s}\left(\mathbf{y}\left(\kappa_{i}, \boldsymbol{\mu}\right)\right)-\mathbf{s}_{m}\left(\kappa_{i}\right)\right\|_{2}^{2}+\frac{\beta}{2}\|\boldsymbol{\mu}\|_{2}^{2} \\
\text { s.t. } \quad\left(\mathbf{K}(\boldsymbol{\mu})-\kappa_{i}^{2} \mathbf{M}(\boldsymbol{\mu})\right) \mathbf{w}\left(\kappa_{i}, \boldsymbol{\mu}\right)=\mathbf{f}\left(\kappa_{i}, \boldsymbol{\mu}\right), \\
\quad \mathbf{y}\left(\kappa_{i}, \boldsymbol{\mu}\right)=\mathbf{G}\left(\kappa_{i}, \boldsymbol{\mu}\right) \mathbf{w}\left(\kappa_{i}, \boldsymbol{\mu}\right), \quad i=1, \cdots, N_{\kappa},
\end{aligned}
$$

where $\frac{\beta}{2}\|\boldsymbol{\mu}\|_{2}^{2}$ is a Tikhonov regularization term and $\left\{\alpha_{i}\right\}_{i=1}^{N_{\kappa}}, \beta$ are appropriate positive weights [28].

\subsubsection{Model order reduction}

For each different wavenumber, the solution of the discrete direct problem (14) - which is required for the evaluation of the discretized PDE constraint - requires a CPU-intensive re-factorization of the matrix on the left-hand side. Hence, to enable an efficient frequency sweep analysis for a given shape configuration of the mockup submarine defined by $\boldsymbol{\mu}$, a frequency sweep ROM characterized by the reduced matrices $\mathbf{K}_{r}(\boldsymbol{\mu}), \mathbf{M}_{r}(\boldsymbol{\mu}), \mathbf{f}_{r}(\kappa, \boldsymbol{\mu})$ and $\mathbf{G}_{r}(\kappa, \boldsymbol{\mu})$ is built using the derivative-based Galerkin projection (DGP) framework described in [20,29]. In this framework - which is based on the moment-matching method, also known as an interpolatory model order reduction method $-N_{\partial}^{\text {DGP }}$ derivatives of the solution with respect to the wavenumber $\kappa$ are first computed by solving (14) for recursively constructed right-hand sides at $N_{\kappa}^{\text {DGP }}$ interpolating wavenumbers $[20,29]$. Then, these derivatives are orthogonalized to achieve numerical robustness, and used to construct an approximation subspace of dimension $k=N_{\kappa}^{\mathrm{DGP}} N_{\partial}^{\mathrm{DGP}}$. The Galerkin projection of (14) onto this subspace leads to the reduced matrices $\mathbf{K}_{r}(\boldsymbol{\mu}), \mathbf{M}_{r}(\boldsymbol{\mu}), \mathbf{f}_{r}(\kappa, \boldsymbol{\mu})$ and $\mathbf{G}_{r}(\kappa, \boldsymbol{\mu})$, and therefore to the desired ROM. Note that this ROM is constructed for a given value of the shape parameters $\boldsymbol{\mu}$. It is suitable for a fast frequency sweep analysis - that is, an analysis where the wavenumber $\kappa$ (and not the vector of shape parameters $\boldsymbol{\mu}$ ) is varied.

As an example, Figure 2 depicts a triangulation of the computational domain (left) with the elements in the PML layer shown in cyan; the real part of the solution for $\kappa=20, L^{s}=1$, and $t^{s}=0.2$ is shown on the right.

To enable the solution of the parametric inverse acoustic scattering problem considered here, a database of frequency sweep ROMs $\mathcal{D} \mathcal{B}$ is constructed offline by sampling the shape parameter space $\mathcal{D}$, using an adaptive approach that will be described below. For each different sampled value of the parameter vector $\boldsymbol{\mu}$ : a different mesh is used to triangulate the computational domain; $N_{\partial}^{\text {DGP }}=8$ (including the 0 -th derivative) derivatives are computed for the same $N_{\kappa}^{\text {DGP }}=2$ interpolating frequencies $\kappa \in\{10,20\}$, leading to a frequency sweep ROM of dimension $k=16$; the $\mathrm{QoI}$ (16) is evaluated at $N_{0}=360$ points that are equidistributed on the circle $S^{1}$.

\subsubsection{Effect of ROM consistency}

Before constructing the main ROM database of interest and using it to illustrate the solution of an inverse acoustic scattering problem in real-time, a first set of numerical experiments is performed here using a very simple database of ROMs to highlight the importance of enforcing ROM consistency. For this purpose, a 

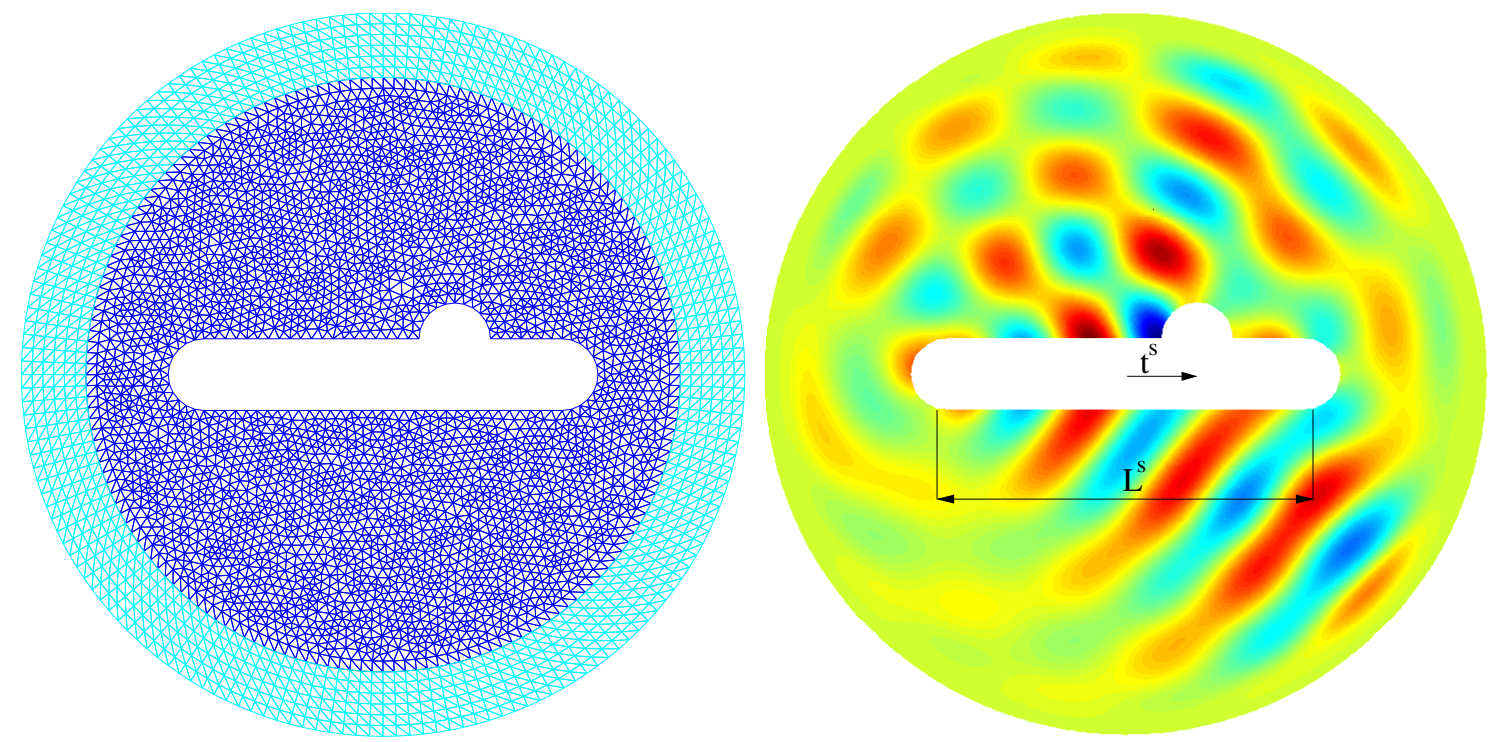

Figure 2 Computational mesh, definitions of $t^{s}$ and $L^{s}$, and real part of the pressure solution for $\kappa=20$.

small database with $N_{\mathrm{DB}}=4$ frequency sweep ROMs is constructed for sampled values of the parameter vector $\boldsymbol{\mu}=\left(\mu_{1}, \mu_{2}\right)$ that are reported in Table 1, together with the dimensions (number of dofs) of the underlying HDMs (note that each HDM has a different number of dofs). Consistency among all computed ROMs is enforced using the procedure for arbitrary meshes presented in Section 3.3. A copy of each ROM before the consistency transformation is applied is also kept in the database for the sole purpose of contrasting the numerical predictions performed using both sets of consistent and not necessarily consistent (referred to in the remainder of this paper as inconsistent) ROMs. Specifically, the far-field patterns are predicted in each case for $\kappa=14$ at the unsampled parameter vector $\boldsymbol{\mu}^{\star}=[0.9625,0.1125]$, by interpolating the pre-computed reduced operators as follows:

- Bilinear interpolation is performed in each case.

- The reduced operators $\operatorname{Re}\left(\mathbf{M}_{r}(\cdot)\right), \operatorname{Im}\left(\mathbf{M}_{r}(\cdot)\right)$ and $\operatorname{Re}\left(\mathbf{K}_{r}(\cdot)\right)$ are interpolated using the approach based on the Cholesky decomposition outlined in Section 4.3.

- The reduced operator $\operatorname{Im}\left(\mathbf{K}_{r}(\cdot)\right)$ is interpolated on the manifold of symmetric matrices of dimension 16.

- The reduced operators $\left\{\mathbf{f}_{r}\left(\kappa_{i}, \cdot\right), \mathbf{G}_{r}\left(\kappa_{i}, \cdot\right)\right\}_{i=1}^{N_{\kappa}}$ are interpolated on the manifold of rectangular complex matrices.

\begin{tabular}{|c|c|c|c|}
\hline $\mathcal{D B}$ point & $\mu_{1}$ & $\mu_{2}$ & $N(\boldsymbol{\mu})$ \\
\hline 1 & 0.95 & 0.1 & 41,235 \\
\hline 2 & 0.975 & 0.1 & 40,965 \\
\hline 3 & 0.95 & 0.125 & 41,424 \\
\hline 4 & 0.975 & 0.125 & 40,929 \\
\hline
\end{tabular}

Table 1 Database of ROMs of dimension 16, and dimensions of underlying HDMs.

Figure 3 reports the computed far-field patterns (16) for $\kappa=14$ at $\boldsymbol{\mu}^{\star}=[0.9625,0.1125]$, using: (a) the HDM, and (b) the application of the ROM interpolation approach outlined above to the sets of consistent and 
inconsistent pre-computed of ROMs. It shows that whereas the computations performed using the database of consistent ROMs are very accurate, the prediction using the database of the inconsistent ROMs has large errors.

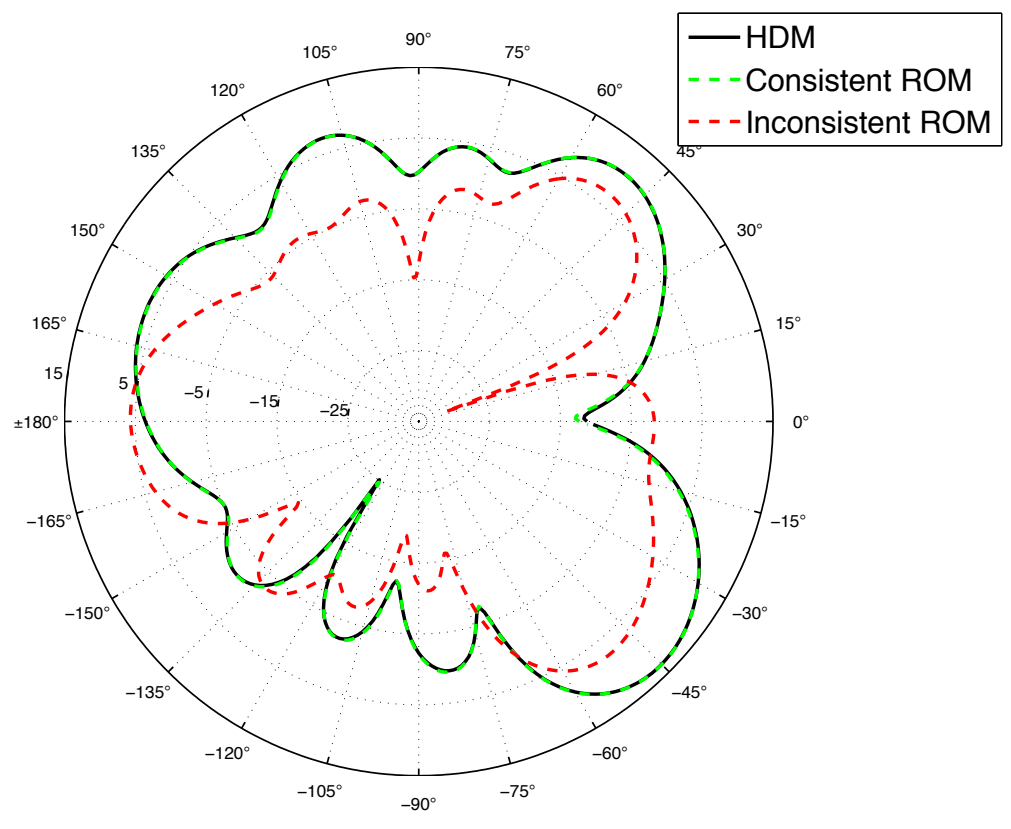

Figure 3 Comparison of the far-field pattern predictions obtained by interpolating consistent and inconsistent pre-computed ROMs.

\subsubsection{Real-time solution of a parametric inverse acoustic scattering problem}

Next, a larger database of ROMs is constructed using an adaptive, goal-oriented sampling procedure that can be described as follows. First, the database is initialized by ROMs built in the vertices of a hypercube (in this case a square) spanning the parameter space. Then, an error of the QoI is computed at the center of the hypercube by interpolating the pre-computed ROMs, and comparing the obtained result to its counterpart obtained using the HDM at the same parameter space point. If the error is found to be above a specified threshold, the hypercube is refined, and the procedure described so far is repeated recursively. Although developed independently from the adaptive sampling procedure discussed at length in [30] and in a different context, the adaptive sampling approach outlined above is related to that presented in [30] (for other adaptive parameter domain partitioning and ROM refinement approaches, see e.g. [31]). The underlying error can be associated with an output of interest, such as the far-field pattern, leading to a goal-oriented approach. Here, the shape $\widehat{\boldsymbol{\mu}}$ is determined by solving the following reduced inverse acoustic scattering problem

$$
\begin{aligned}
\min _{\boldsymbol{\mu} \in \mathcal{D}} \sum_{i=1}^{N_{\kappa}} \alpha_{i}\left\|\mathbf{s}\left(\mathbf{y}_{r}\left(\kappa_{i}, \boldsymbol{\mu}\right)\right)-\mathbf{s}_{m}\left(\kappa_{i}\right)\right\|_{2}^{2}+\frac{\beta}{2}\|\boldsymbol{\mu}\|_{2}^{2} \\
\text { s.t. } \quad\left(\mathbf{K}_{r}(\boldsymbol{\mu})-\kappa_{i}^{2} \mathbf{M}_{r}(\boldsymbol{\mu})\right) \mathbf{q}\left(\kappa_{i}, \boldsymbol{\mu}\right)=\mathbf{f}_{r}\left(\kappa_{i}, \boldsymbol{\mu}\right), \\
\quad \mathbf{y}_{r}\left(\kappa_{i}, \boldsymbol{\mu}\right)=\mathbf{G}_{r}\left(\kappa_{i}, \boldsymbol{\mu}\right) \mathbf{q}\left(\kappa_{i}, \boldsymbol{\mu}\right), \quad i=1, \cdots, N_{\kappa},
\end{aligned}
$$

using simulated annealing (specifically, the simUlannEALBND Matlab routine with its default options, and a starting point in the lower left corner of the bounding box given by the current hypercube in the training 
stage, or the parameter domain bounds in the online stage) is defined as the QoI. The corresponding error measure is defined as

$$
\text { Error }=\left\|\frac{\widehat{\boldsymbol{\mu}}-\boldsymbol{\mu}^{\star}}{\boldsymbol{\mu}_{\max }-\boldsymbol{\mu}_{\min }}\right\|_{\infty}
$$

where $\boldsymbol{\mu}^{\star}$ denotes the target shape configuration, $\boldsymbol{\mu}_{\min }$ and $\boldsymbol{\mu}_{\max }$ the lower and upper bounds for each shape parameter, respectively, and the ratio in Error is computed entry-by-entry.

Figure 4 (left) graphically depicts the constructed database for the relative error tolerance of $5 \%$, and the parameter domain $\mathcal{D}=[0.9,1] \times[0.1,0.2]$. The reader can observe that this domain has been sampled in $N_{\mathrm{DB}}=22$ points. The training errors obtained at each sampling refinement iteration are reported in Figure 4 (right). After the second refinement iteration, all errors are found to be below the threshold of $5 \%$.

To validate the global accuracy of the constructed ROM database, 289 unsampled target shape configurations $\left(\mu_{1}, \mu_{2}\right)$ are queried in $\mathcal{D}$, and the inverse acoustic scattering problems at these shape configurations are solved using the database of ROMs. The obtained errors and their distribution are reported in Figure 5. respectively. All errors can be observed to be below the given threshold, confirming the validity of the training procedure.
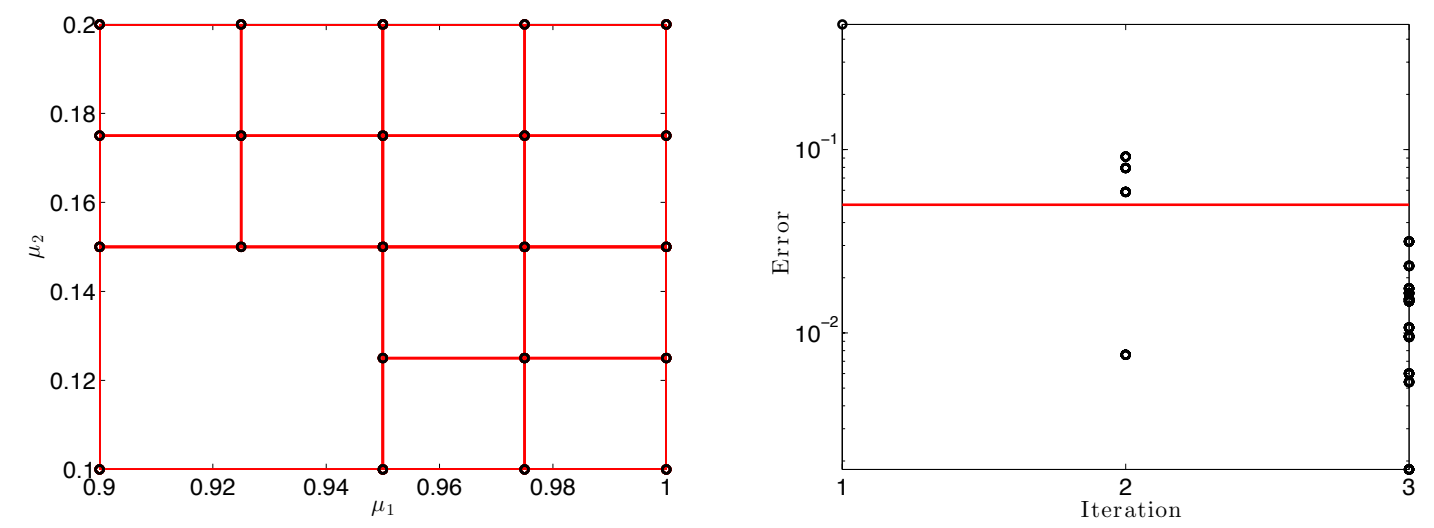

Figure 4 Database of pre-computed ROMs $\mathcal{D B}$ constructed using a goal-oriented adaptive sampling procedure (left). Training errors at each iteration of the goal-oriented adaptive sampling procedure (right).
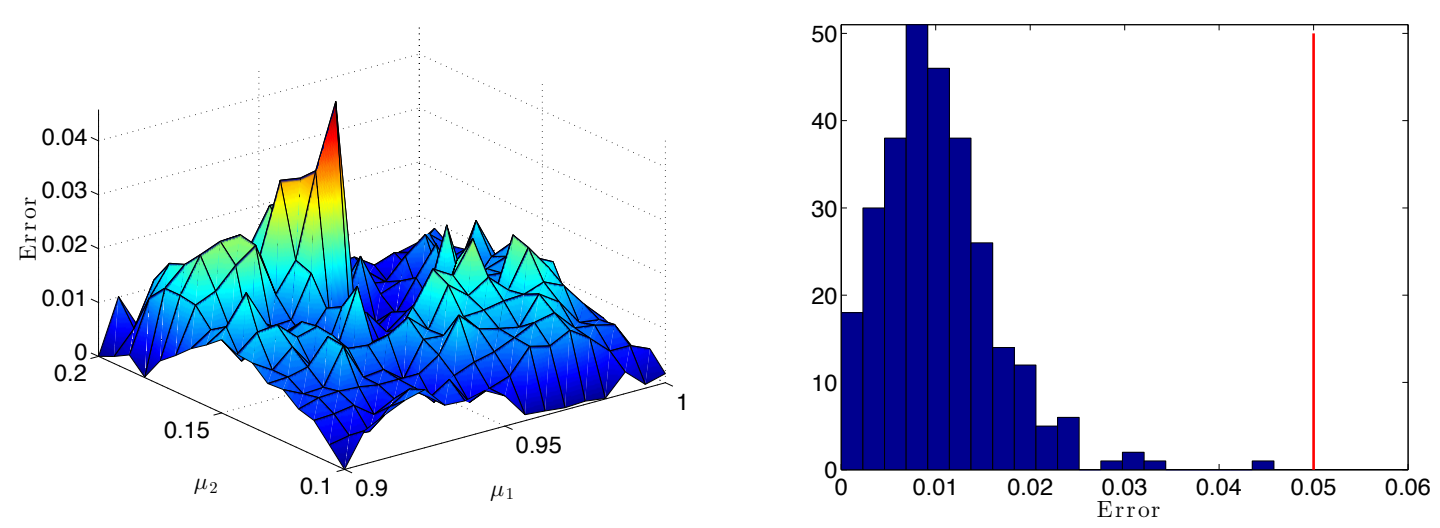

Figure 5 Prediction errors (left) and their distribution (right) obtained for the solution of 289 inverse acoustic scattering problems.

Finally, for a Matlab implementation of the computations described above, the wall-clock timings associated with the solution of a single instance of the parametric inverse acoustic scattering problem using both 
the ROM database strategy and the appropriate HDM are reported in Table 2. These timings highlight an impressive speedup of 270 due to the proposed ROM database approach. For a typical function call within the optimization procedure, the speedup due to model reduction is found to be equal to 207.

\begin{tabular}{|c|c|c|c|c|}
\hline Computational approach & Error & $\begin{array}{c}\text { Number of } \\
\text { function calls }\end{array}$ & $\begin{array}{c}\text { Online optimization } \\
\text { wall-clock time }\end{array}$ & Speed-up factor \\
\hline \hline HDM & $8 \times 10^{-4}$ & 1530 & $1 \mathrm{~h} 30 \mathrm{~min}$ & 1 \\
\hline ROM database & 0.02 & 1176 & $20.1 \mathrm{~s}$ & 270 \\
\hline
\end{tabular}

Table 2 Wall-clock timings associated with the solution of the inverse acoustic scattering problem using the appropriate HDM and the database of ROMs.

REMARK. The cost of adaptive parameter sampling on a hypercube can be expected to grow rapidly with the number of parameters. This issue - which theoretically holds for many sampling approaches may turn out to be in practice a limitation of the chosen sampling procedure. However, it is not a direct limitation of the proposed approach for consistent ROM interpolation.

\subsection{Flutter analysis}

\subsubsection{Problem formulation}

The flutter analysis considered here is for a wing-store system in the subsonic, transonic, and supersonic regimes. This system is fully described in [32]. It is parameterized by: the fuel fill level $f$ (in percentage) inside the store, which affects primarily the mass of the system, its hydroelastic effects [32] and therefore, its aeroelastic behavior; and the free-stream Mach number $M_{\infty}$, which affects primarily the flow around this system and consequently its aeroelastic behavior.

The structural subsystem is a second-order LTIP of the form (3). It is represented here by a sufficiently detailed finite element model. For each different fuel fill level, a new structural mesh is generated inside the store and therefore a new structural HDM is constructed. The hydroelastic effects are modeled using the added mass approach [32], so that the dimension of the resulting linear structural HDM is independent from $f$. In this case, it is set to $N^{(s)}=6,834$.

The fluid subsystem is modeled using the linearized Euler equations and an inviscid CFD grid with roughly 80,000 grid points. Specifically, the governing Euler equations are semi-discretized by a finite volume method based on a second-order accurate linear flux reconstruction, and discretized using the second-order accurate implicit three point backward difference scheme. For each operating point $\boldsymbol{\mu}=\left(M_{\infty}, f\right)$, the linearization is performed around the steady-state aeroelastic equilibrium solution associated with these two parameters. This results in a first-order LTIP fluid subsystem of the form $(1)$ with $N^{(f)} \approx 400,000$ dofs.

A finite element model of the structural subsystem and the CFD surface grid are graphically depicted in Figure 6 .

Given the subsystem computational models outlined above, the coupled linearized aeroelastic HDM can be described by the following system of equations

$$
\begin{aligned}
\widetilde{\mathbf{A}} \frac{d \mathbf{w}}{d t}(t)+\widetilde{\mathbf{H}} \mathbf{w}(t)+\widetilde{\mathbf{R}} \frac{d \mathbf{u}}{d t}(t)+\widetilde{\mathbf{G}} \mathbf{u}(t) & =\mathbf{0} \\
\widetilde{\mathbf{M}} \frac{d^{2} \mathbf{u}}{d t^{2}}(t)+\widetilde{\mathbf{K}} \mathbf{u}(t) & =\widetilde{\mathbf{P}} \mathbf{w}(t),
\end{aligned}
$$

where $\mathbf{w}$ denotes the vector of fluid dofs, $\mathbf{u}$ is the vector of structural dofs, $\widetilde{\mathbf{A}}$ denotes the diagonal matrix of fluid cell volumes, $\widetilde{\mathbf{H}}$ the Jacobian matrix of the finite volume fluxes, and $\widetilde{\mathbf{R}}, \widetilde{\mathbf{G}}$ and $\widetilde{\mathbf{P}}$ are linearized fluid-structure coupling operators (see [33] for further details). $\widetilde{\mathbf{M}}$ and $\widetilde{\mathbf{K}}$ are the finite element mass and stiffness matrices of the structural subsystem.

The above system of equations can be re-written in a first-order LTIP aeroelastic system as follows

$$
\mathbf{E}(\boldsymbol{\mu}) \frac{d \mathbf{z}}{d t}(t)=\mathbf{A}(\boldsymbol{\mu}) \mathbf{z}(t)
$$




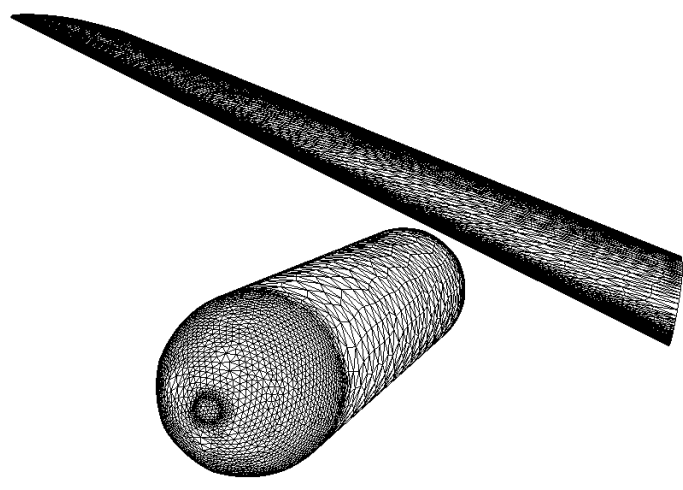

(a) CFD surface grid

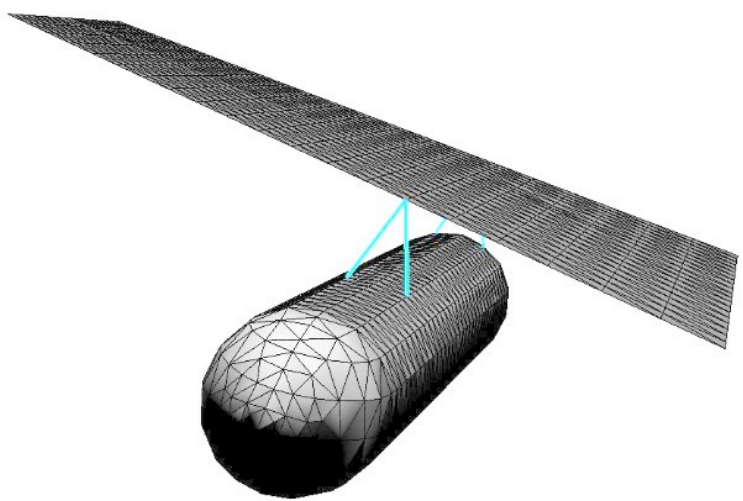

(b) Finite element structural model

Figure 6 High-dimensional aeroelastic model of a wing-store configuration.

where

$$
\mathbf{E}=\left[\begin{array}{ccc}
\widetilde{\mathbf{A}} & 0 & 0 \\
0 & \widetilde{\mathbf{M}} & 0 \\
0 & 0 & \mathbf{I}
\end{array}\right], \quad \mathbf{A}=\left[\begin{array}{ccc}
-\widetilde{\mathbf{H}} & -\widetilde{\mathbf{R}} & -\widetilde{\mathbf{G}} \\
\widetilde{\mathbf{P}} & 0 & -\widetilde{\mathbf{K}} \\
0 & \mathbf{I} & 0
\end{array}\right], \quad \text { and } \quad \mathbf{z}=\left[\begin{array}{c}
\mathbf{w} \\
\dot{\mathbf{u}} \\
\mathbf{u}
\end{array}\right]
$$

The parameter space of interest is set to $\left(M_{\infty}, f\right) \in \mathcal{D}=[0.6,1.1] \times[0,100]$. For each operating point within this parameter space, the critical pressure values $p_{\infty}^{\mathrm{cr}}$ and critical velocity values $V_{\infty}^{\mathrm{cr}}$ associated with the onset of flutter are sought, and the QoI is set to the flutter speed index (FSI) at this operating point. The FSI is computed as

$$
\mathrm{FSI}=\frac{V_{\infty}^{\mathrm{cr}}}{b_{s} \omega_{\alpha} \sqrt{\bar{\mu}}},
$$

where $b_{s}$ is the semi-chord of the wing at its root, $\omega_{\alpha}$ is the first dry torsional mode of the wing-store structural system and $\bar{\mu}$ is the mass ratio as defined in $[34,32]$.

\subsubsection{Model order reduction and real-time flutter analysis}

130 operating points corresponding to 26 different free-stream Mach number values and 5 different fuel fill levels are queried in the domain $\left(M_{\infty}, f\right) \in \mathcal{D}=[0.6,1.1] \times[0,100] \%$. First, the QoIs are computed at these points using the corresponding coupled aeroelastic HDMs. These are built in this case using the same structural and fluid meshes. The obtained results are reported in the left portion of Figure 7 . Note that the typical flutter dip can be observed in this figure at $M_{\infty} \approx 0.96$.

Next, $N_{\mathrm{DB}}=21$ operating points are sampled on the lattice $\left(M_{\infty}, f\right) \in\{0.6,0.75,0.9,0.95,1.0,1.05,1.1\} \times$ $\{0,50,100\}$, and linear aeroelastic ROMs are constructed at these points offline, using the fluid and structural model order reduction techniques described in [11]. Specifically, for each operating point, a structural modal ROM of dimension $k^{(s)}=4$ and a POD-based fluid ROM of dimension $k^{(f)}=15$ are constructed and coupled as described in [11]. Given that in this case all underlying HDMs share the same fluid and structural meshes, consistency between all ROMs is enforced using the approach presented in Section 3.2. Then, the transformed ROMs are stored in a database $\mathcal{D} \mathcal{B}$. The QoIs computed at these 21 sampled points are reported in the right portion of Figure 7.

Then, the 21 pre-computed linear aeroelastic ROMs are organized in $N_{s}=3$ different databases (or sub-databases of $\mathcal{D B}$ ), based on the flow physics of the problem. The first of these covers the subsonic and lower transonic flow regimes $\left(M_{\infty} \in[0.6,0.9]\right)$. The second one covers the upper transonic flow regime 

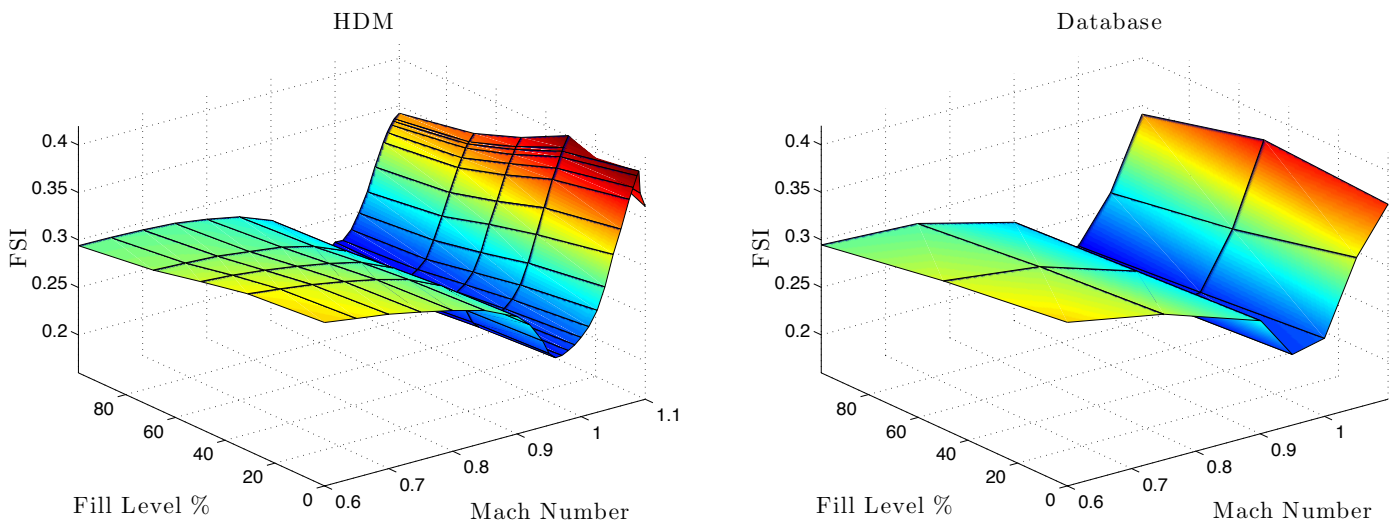

Figure 7 FSI values computed using the appropriate instances of an aeroelastic HDM (left) and a database of linear aeroelastic ROMs (right).

$\left(M_{\infty} \in[0.9,1.0]\right)$ and the third one the supersonic flow regime $\left(M_{\infty} \in[1.0,1.1]\right)$. All three ROM databases are graphically depicted in Figure 8.

In preparation for the online computations, the heuristic developed in [15] and mentioned in Section 4.3 is applied to select the matrix manifold on which to perform the interpolation of reduced linearized fluid operators. Indeed, for these operators, interpolation can be performed on the matrix manifold GL $\left(k^{(f)}\right)$ of non-singular matrices of size $k^{(f)}$, or on $\mathbb{R}^{k^{(f)} \times k^{(f)}}$. In anticipation of piecewise-linear interpolation in the $M_{\infty}$ "direction" and cubic spline interpolation in the $f$ direction, this heuristic is applied in six different regions of the parametric space, as reported in Table 3. The reader can observe that the matrix manifold $\mathrm{GL}\left(k^{(f)}\right)$ is chosen by this heuristic in two regions, while the matrix manifold $\mathbb{R}^{k^{(f)} \times k^{(f)}}$ is chosen in four regions.

\begin{tabular}{|c|c|c|c|c|c|c|}
\hline & \multicolumn{2}{|c|}{ Database 1 } & \multicolumn{2}{c|}{ Database 2 } & \multicolumn{2}{c|}{ Database 3 } \\
\hline$M_{\infty} \in$ & {$[0.6,0.75]$} & {$[0.75,0.9]$} & {$[0.9,0.95]$} & {$[0.95,1]$} & {$[1,1.05]$} & {$[1.05,1.1]$} \\
\hline GL $\left(k^{(f)}\right)$ & 0.89 & 0.87 & 0.93 & 0.93 & 0.76 & 0.86 \\
\hline $\mathbb{R}^{k^{(f)} \times k^{(f)}}$ & 0.84 & 0.86 & 0.98 & 0.87 & 0.78 & 0.82 \\
\hline Matrix manifold & $\mathbb{R}^{k^{(f)} \times k^{(f)}}$ & $\mathbb{R}^{k^{(f)} \times k^{(f)}}$ & $\mathrm{GL}\left(k^{(f)}\right)$ & $\mathbb{R}^{k^{(f)} \times k^{(f)}}$ & GL $\left(k^{(f)}\right)$ & $\mathbb{R}^{k^{(f)} \times k^{(f)}}$ \\
\hline
\end{tabular}

Table 3 Nonlinearity indicator and chosen matrix manifold.

Finally, the proposed comprehensive approach for real-time computations based on a database of parametric, linear, projection-based ROMs is applied to the computation of the QoIs at the same 130 queried operating points. However, before the accuracy obtained for these QoIs is analyzed, that obtained for the intermediate quantities that are the interpolated structural operators is assessed. To this effect, Figure 9 contrasts the eigenvalues of these operators - that is, the natural, dry frequencies of the aeroelastic system at all queried fuel fill levels - with their counterparts obtained directly from the instances of the HDM at these fuel fill levels. A good agreement between these two sets of results can be observed, even for the unsampled fuel fill levels.

The obtained QoIs themselves are plotted in Figure 10, where they are also compared to their counterparts predicted using a response surface estimation (RSE) based on a direct bicubic spline interpolation of the pre-computed QoIs. Recalling that the reference (HDM) values of these QoIs are reported in the left portion of Figure 7, the reader can observe that while the numerical predictions performed using the ROM database approach developed in this paper are very accurate, those obtained using RSE are much less accurate, especially near the transonic dip and in the supersonic regime. In particular, the left portion of Figure 10 


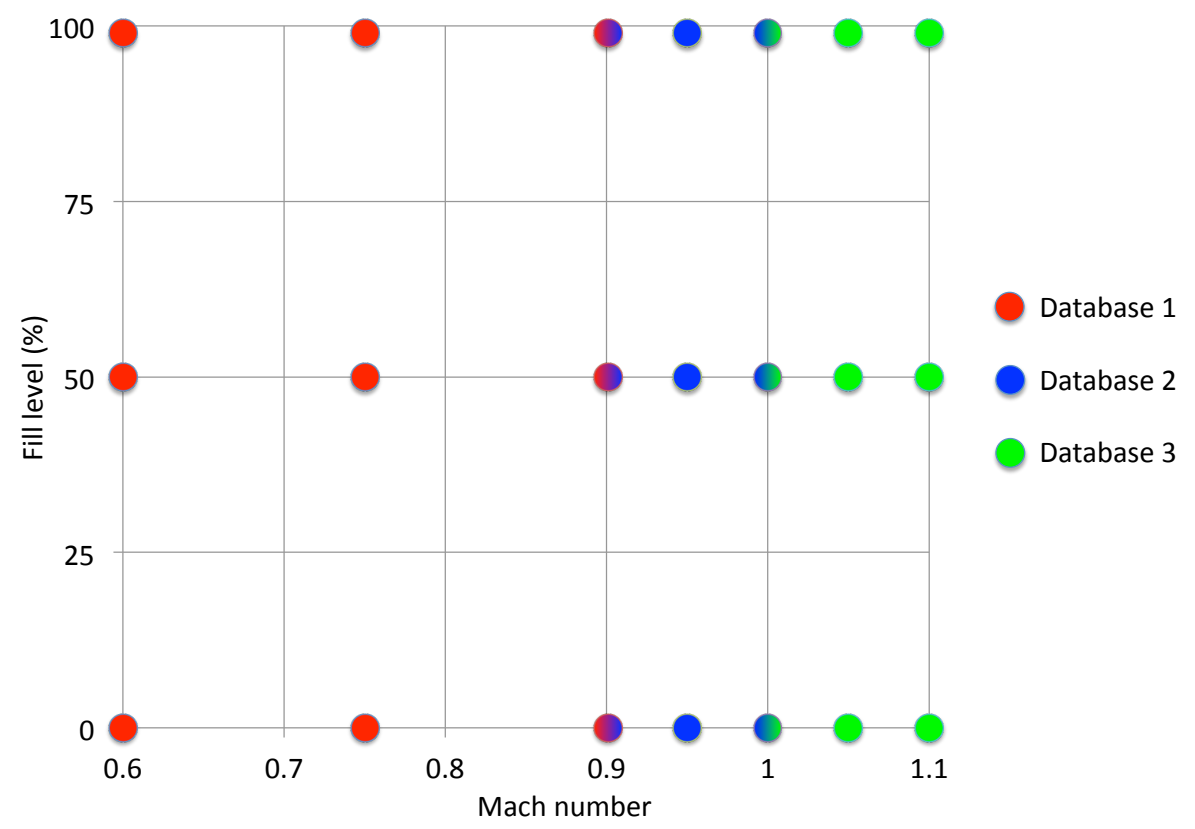

Figure 8 Pre-computed databases $\left\{\mathcal{D B}_{s}\right\}_{s=1}^{3}$ of linear aeroelastic ROMs.

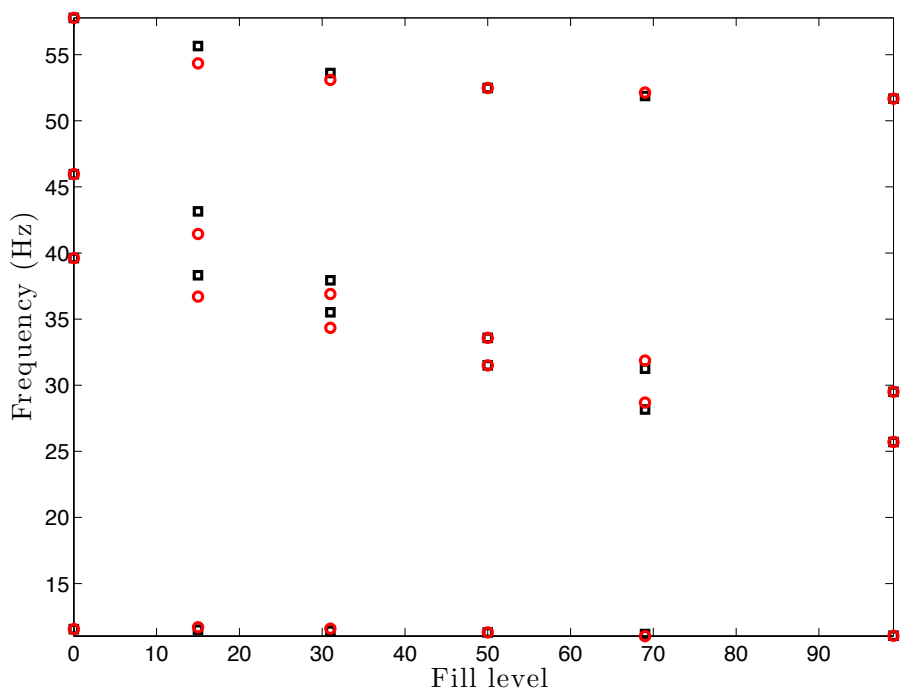

Figure 9 Eigenvalues of the structural subsystem computed at all queried fuel fill levels using the appropriate instances of the HDM $(\square)$ and the interpolated ROMs (o). 
reveals that RSE does not predict the correct variation of the FSI at supersonic speeds and low fuel fill levels. On the other hand, the right portion of this same figure shows that the proposed approach based on a database of linear ROMs captures remarkably well the complex behavior of the FSI in the supersonic flow regime at low fuel fill levels. It underlines the superiority of the proposed idea of interpolating the operators underlying a QoI to compute the desired QoI at a queried but unsampled point, rather than interpolating directly the pre-computed QoIs themselves to obtain a value of the QoI at this point.
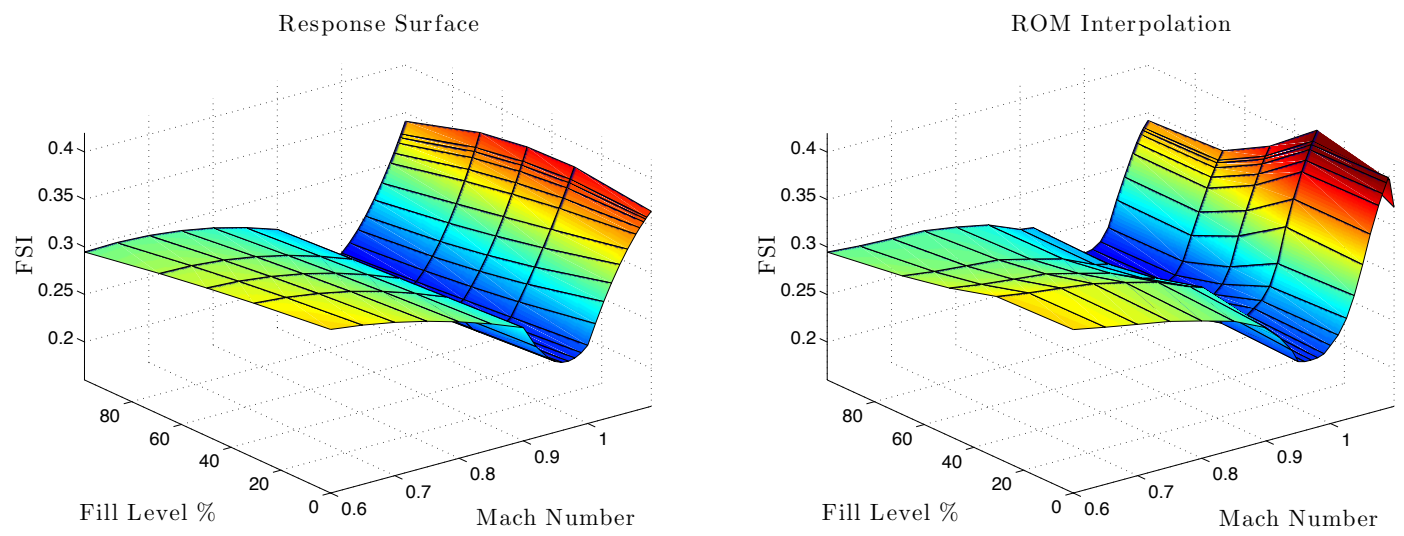

Figure 10 FSI values computed using response surface estimation (left) and ROM interpolation (right).

The wall-clock performance results associated with the numerical prediction of the QoIs at all 130 queried operating points using all three considered computational approaches and a desktop computer are reported in Table 4. These results demonstrate the real-time capability of the proposed computational approach, as they highlight its ability to perform all 130 predictions not only accurately, but in 31 seconds only.

\begin{tabular}{|c|c|c|c|c|}
\hline Computational approach & \multicolumn{2}{|c|}{ Offline phase } & \multicolumn{2}{c|}{ Online phase } \\
\hline & Wall-clock time (s) & $\begin{array}{l}\text { Number of } \\
\text { processors }\end{array}$ & Wall-clock time (s) & $\begin{array}{c}\text { Number of } \\
\text { processors }\end{array}$ \\
\hline HDM & NA & NA & $9,152,000$ & 32 \\
\hline RSE & 28,000 & 32 & 2 & 1 \\
\hline ROM database & 28,000 & 32 & 31 & 1 \\
\hline
\end{tabular}

Table 4 Wall-clock timings associated with the computation on a desktop computer of the FSI values at all 130 queried operating points.

Next, the flutter behavior at supersonic speeds and low fuel fill levels is studied in more detail by predicting the FSI at the additional unsampled fill level of $15 \%$ (see Figure 11). In order to understand the physical phenomenon at play, the eigenvalues of the interpolated aeroelastic ROM matrices are computed for increasing values of the free-stream pressure until flutter is reached. Indeed, by following the wet structural modes in the complex plane, one can determine which structural mode flutters - that is, which structural mode is the first to cross the imaginary axis (see Figure 12 and Figure 13 which focus on four different values of the free-stream Mach number and an empty store). The reader can observe that for $M_{\infty}=1.075$ and $M_{\infty}=1.091$, the first mode is the first to cross the imaginary axis, but for $M_{\infty}=1.092$ and $M_{\infty}=1.1$, the third mode is the first one to flutter. (The considered order of the modes is by the magnitude of their imaginary parts as indicated in Figure 13 where an eigenvalue of the mode that is first to flutter is also circled.) This indicates the presence of a bifurcation phenomenon. This analysis, which cannot be performed using the results of an RSE, also highlights the advantage of model reduction over RSE. (Note that the aeroelastic HDM predicts the same phenomenon - that is, an FSI peak value between $M_{\infty}=1.09$ and $M_{\infty}=1.092$.) 

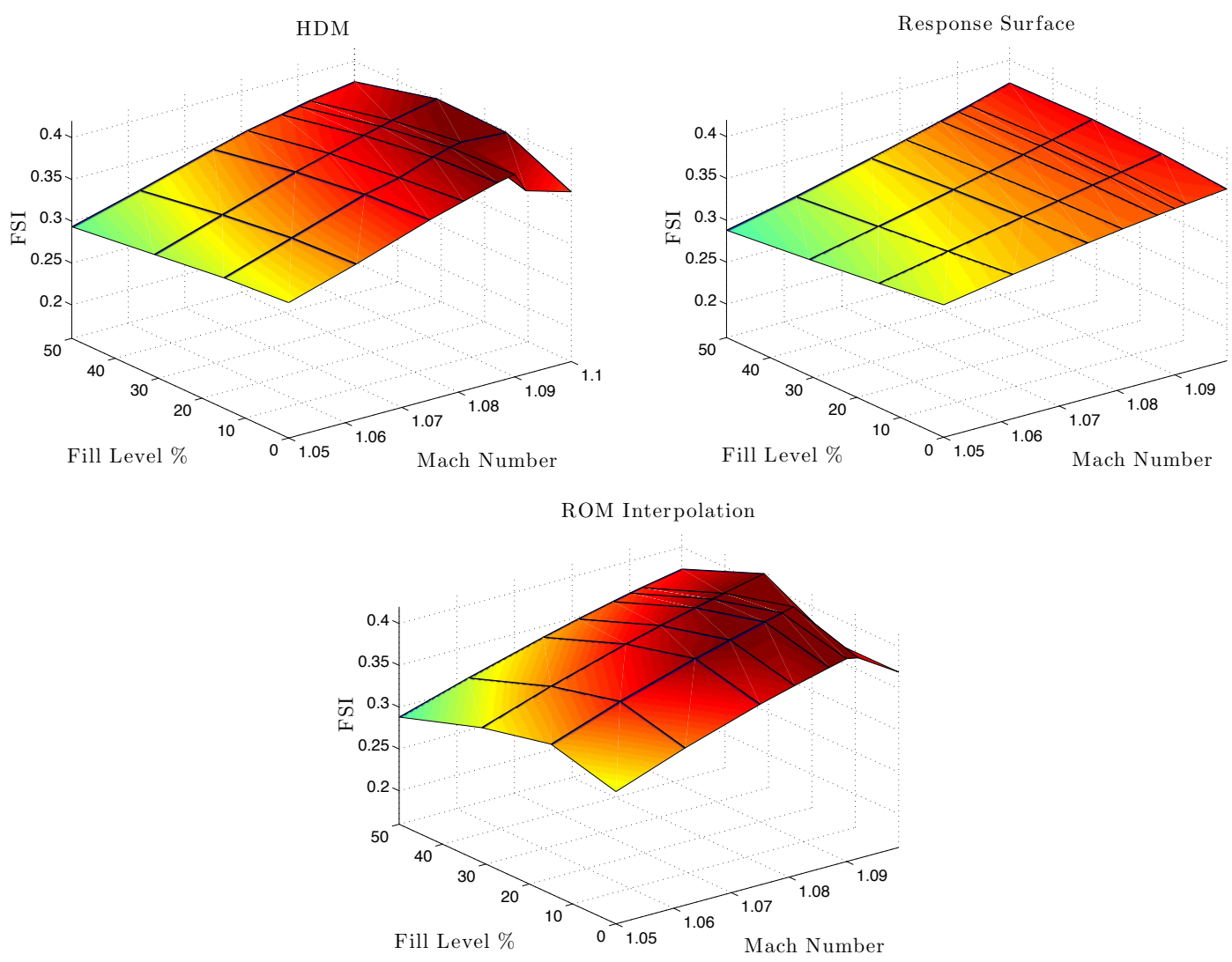

Figure 11 FSI values computed at supersonic speeds and low fuel fill levels using: appropriate instances of the HDM (top left), RSE (top right) and ROM interpolation (bottom). 

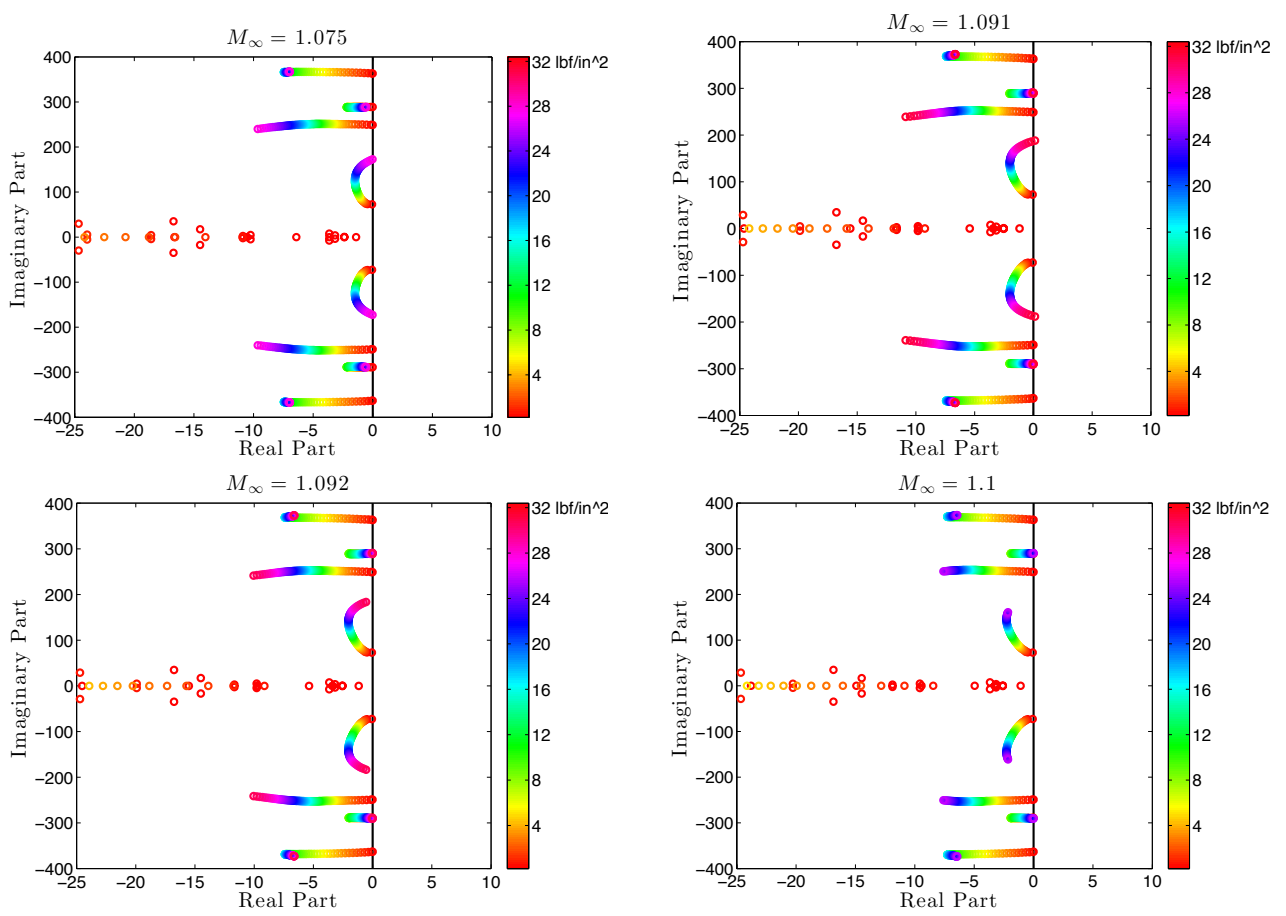

Figure 12 Aeroelastic matrix eigenvalues loci at 0\% fill level for various free-stream Mach numbers.
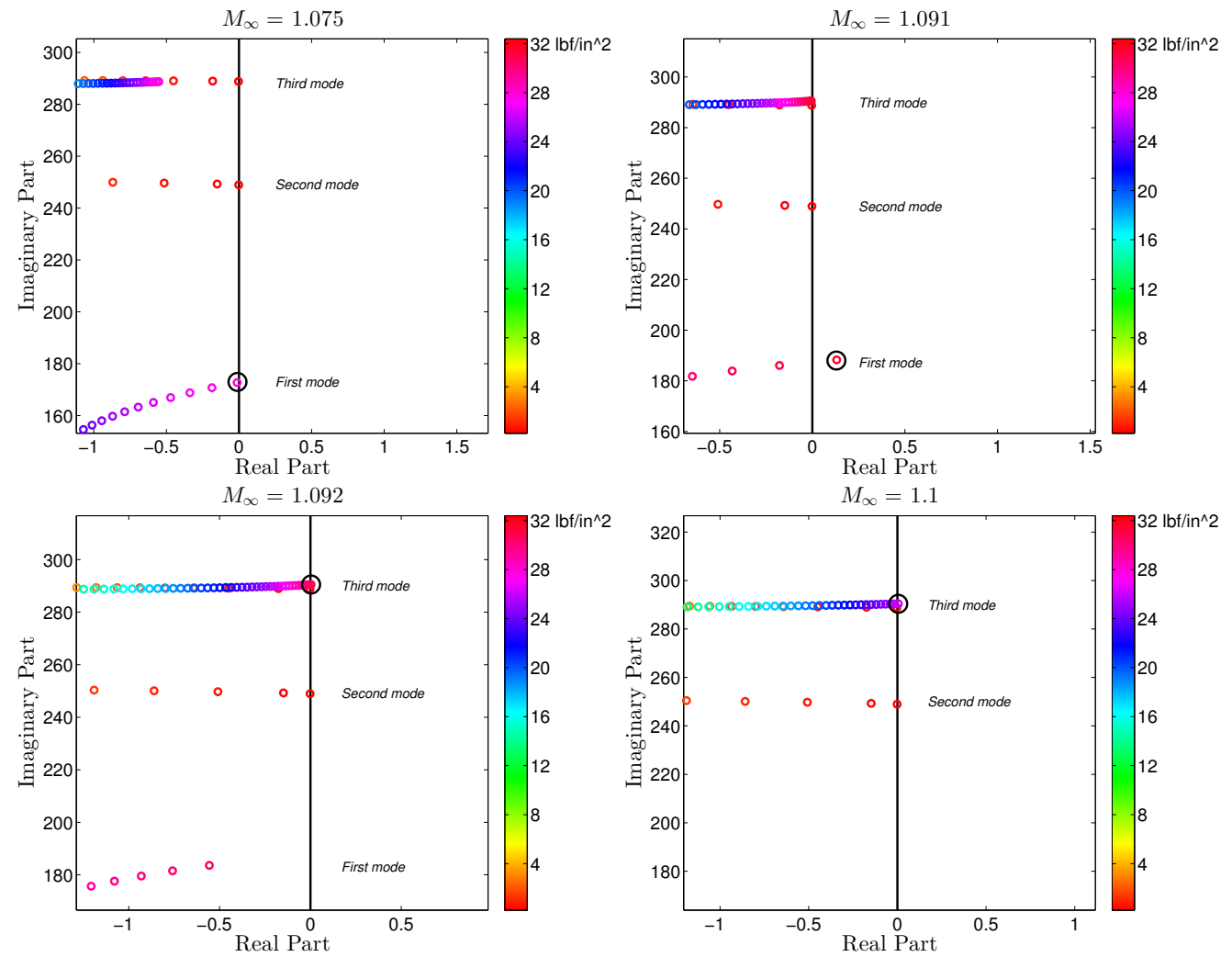

Figure 13 Aeroelastic matrix eigenvalues loci at 0\% fill level for various free-stream Mach numbers (zoomed view). 
Next, the effect of the heuristic developed in [15] for selecting the matrix manifold on which to perform the interpolation of reduced linearized fluid operators is studied by turning off this capability, and repeating all computations discussed above twice: once with all aforementioned interpolations performed on $\mathbb{R}^{k^{(f)} \times k^{(f)}}$, and once on GL( $\left.k^{(f)}\right)$. The obtained numerical results are reported in Figure 14. The comparison of these results with their counterpart displayed in Figure 10 (right) shows that more accurate predictions are obtained when using the heuristic, particularly in the subsonic and transonic regimes.
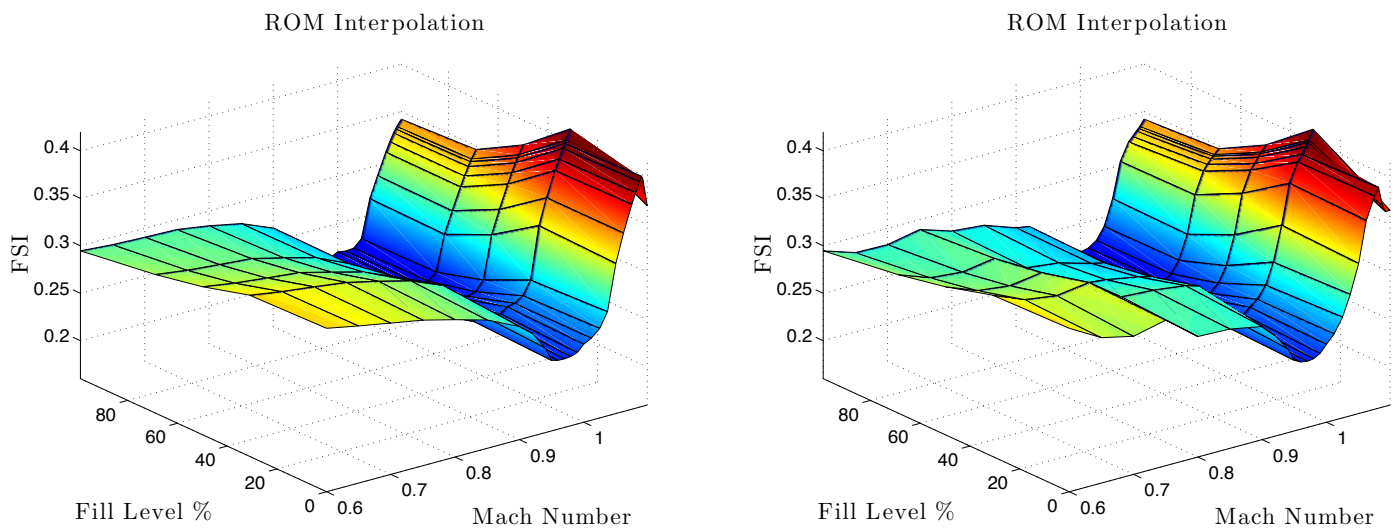

Figure 14 FSI values computed using interpolation of the reduced fluid operators on $\mathbb{R}^{k^{(f)} \times k^{(f)}}$ (left) and on $\operatorname{GL}\left(k^{(f)}\right)$ (right).

The effect of ROM consistency on accuracy is also studied by repeating all aforementioned computations without enforcing ROM consistency for the reduced structural operators, then the reduced fluid operators, then all reduced operators. The obtained results for the QoIs are reported in Figure 15. They show that skipping the enforcement of consistency for either the structural or fluid operators is as detrimental to accuracy as skipping it for all of them.

An interpolated aeroelastic ROM can also be used to predict the displacement at any given location of the wing-store system. To this effect, Figure 16 reports the wing tip displacement time-histories at the transonic dip - that is $M_{\infty}=0.97$ - the fuel fill level of $99 \%$ and three different cruise altitudes, using the appropriate instances of the HDM and the ROM database approach. In general, a good agreement can be observed between the ROM results and their HDM counterparts.

Finally, for this application, the proposed ROM database approach was implemented on an iPhone using two modes of operation, in order to demonstrate its practicality and potential for mobile computing. In the first mode of operation, a value of the fuel fill level is specified by the user and the FSI is computed by the device for a series of free-stream Mach numbers $M_{\infty} \in[0.6,1.1]$. In the second mode of operation, an operating point $\left(M_{\infty}, f\right)$ is specified and the smallest aeroelastic damping ratio is computed for a series of altitudes $h \in[0,40000] \mathrm{ft}$. The first mode of operation is illustrated in Figure 17 .

\section{Conclusions}

A general approach for real-time linear or linearized computations using a database of parametric, projection-based, linear reduced-order models (ROMs) based on arbitrary underlying meshes is presented. In its offline phase, the parameter space is sampled and linear ROMs defined by linear reduced operators are pre-computed at the sampled parameter points, transformed into counterpart ROMs that are consistent among themselves - that is, into ROMs whose underlying generalized coordinates are expressed in the same reference generalized coordinate system - and stored. In its online phase, a linear ROM is constructed at a queried but unsampled parameter point by interpolating the pre-computed linear reduced operators on appropriate matrix manifolds and used to perform the numerical prediction of interest. The results obtained using this computational approach for two different realistic applications, namely, the identification of the 
ROM Interpolation

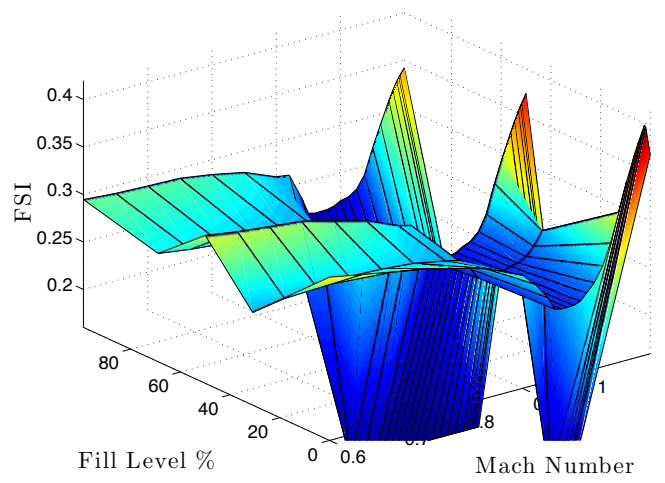

ROM Interpolation

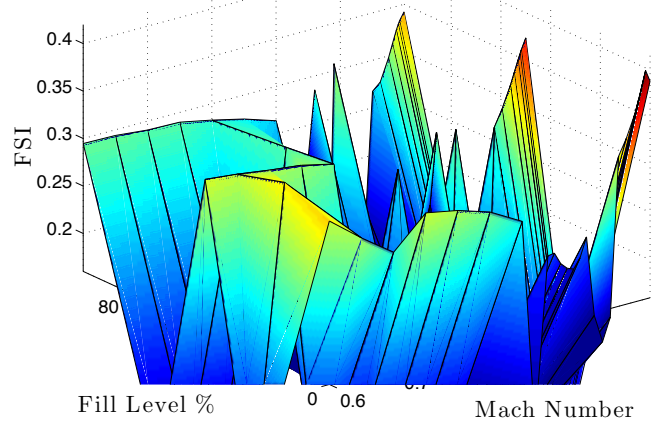

ROM Interpolation

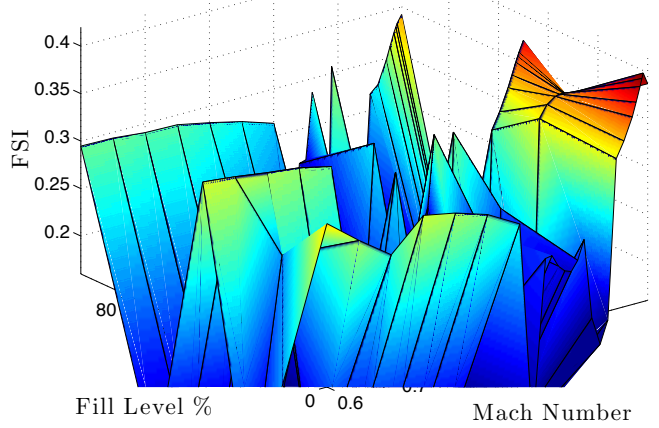

Figure 15 FSI values computed using ROM interpolation without consistency enforcement for the reduced structural operators (top left), the reduced fluid operators (top right) and all reduced operators (bottom). 

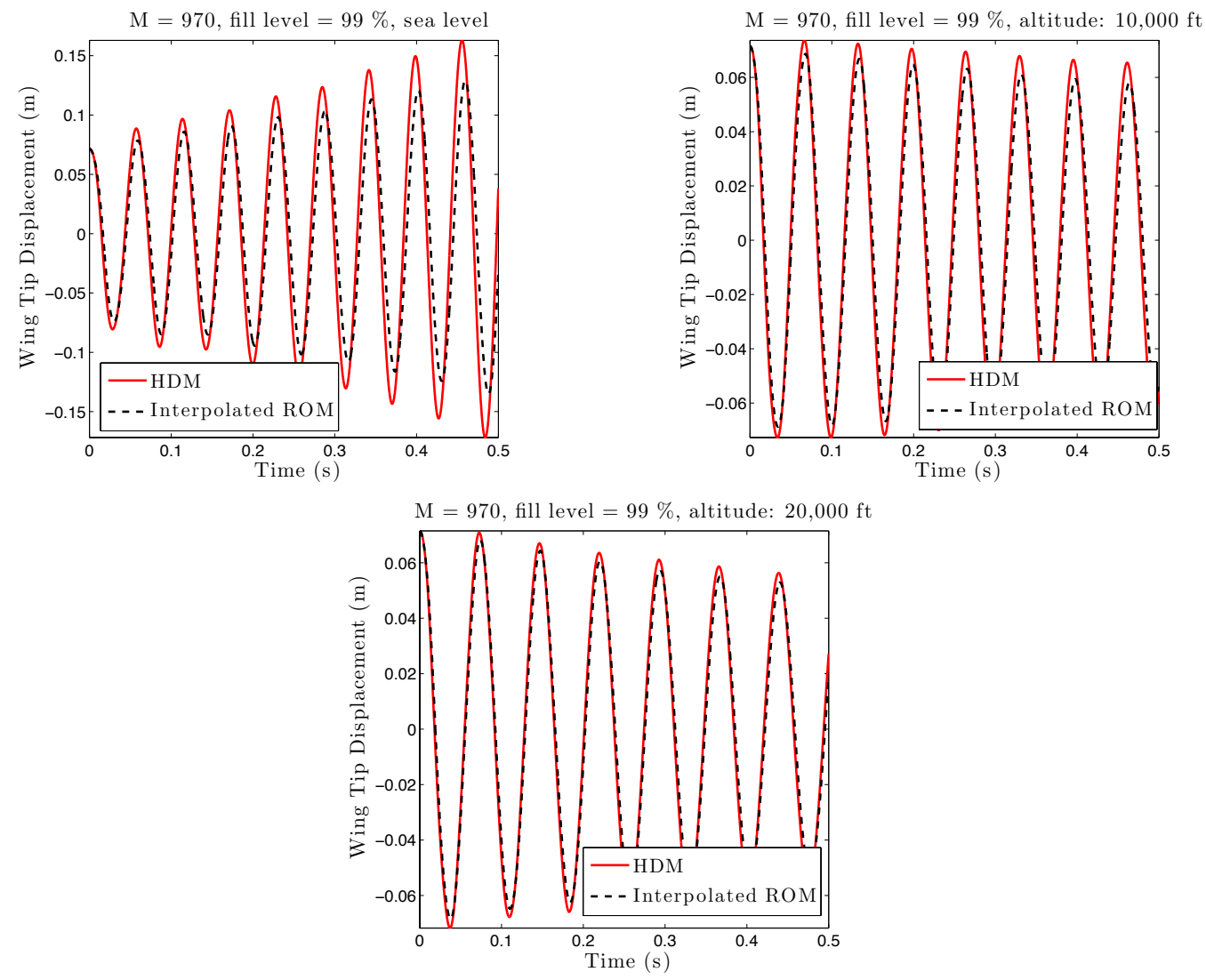

Figure 16 Wing tip displacement computed at $M_{\infty}=0.97,99 \%$ fuel fill level and three different altitudes using the appropriate instances of the HDM and ROM interpolation.

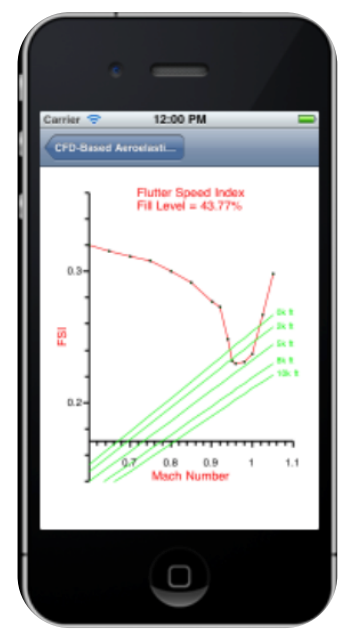

Figure 17 Implementation of the ROM database approach on an iPhone and application to the computation of the FSI for the fuel fill level $f=43.77 \%$. 
parametric shape of an obstacle from the knowledge of far-field patterns it creates when it scatters incident waves, and the flutter analysis of a wing-store configuration for different fuel fill levels and air speeds that span the subsonic, transonic, and supersonic flight regimes, demonstrate the real-time capability of this proposed computational technology. They also illustrate its particular suitability for on-demand computations such as what-if scenarios and embedded computing. These results also underscore the following three other important conclusions. First, ROM consistency must be enforced before ROM interpolation is performed. Second, because a reduced linear operator can belong to multiple matrix manifolds, using some rationale to determine in each region of the parameter space which manifold to use for performing the ROM interpolation is a superior approach to selecting this manifold a priori and using it uniformly in the parameter space. And third, interpolating consistent ROMs to obtain a linear ROM at a queried but unsampled parameter point and using this ROM to compute a quantity of interest (QoI) at this point is a computationally superior approach to computing the QoIs at the sampled parameter points and interpolating them directly.

\section{Acknowledgments}

The authors acknowledge partial support by the Army Research Laboratory through the Army High Performance Computing Research Center under Cooperative Agreement W911NF-07-2-0027, partial support by the Office of Naval Research under Grants no. N00014-11-1-0707 and N00014-14-1-0233, and partial support by The Boeing Company under Contract Sponsor Ref. 45047. This document does not necessarily reflect the position of these institutions, and no official endorsement should be inferred. The authors also thank Mark Potts for his assistance in the implementation of the described computational technology on the iPhone device.

\section{Appendix 1: proof of Theorem 1}

The objective function can be written as

$$
\mathcal{J}_{G}(\mathbf{Q})=\epsilon\left\langle\mathbf{Q}^{T} \mathbf{E}_{r i} \mathbf{Q}, \mathbf{E}_{r}^{0}\right\rangle+\alpha\left\langle\mathbf{Q}^{T} \mathbf{A}_{r i} \mathbf{Q}, \mathbf{A}_{r}^{0}\right\rangle+\langle\mathbf{F}, \mathbf{Q}\rangle,
$$

where $\mathbf{F}=\beta \mathbf{B}_{r i}\left(\mathbf{B}_{r}^{0}\right)^{T}+\gamma \mathbf{C}_{r i}^{T} \mathbf{C}_{r}^{0}$.

The Lagrangian of the optimization problem is then

$$
\begin{aligned}
\mathcal{L}(\mathbf{Q}, \mathbf{S}) & =\mathcal{J}_{G}(\mathbf{Q})+\left\langle\frac{1}{2} \mathbf{S}, \mathbf{I}_{k}-\mathbf{Q}^{T} \mathbf{Q}\right\rangle \\
& =\epsilon\left\langle\mathbf{Q}^{T} \mathbf{E}_{r i} \mathbf{Q}, \mathbf{E}_{r}^{0}\right\rangle+\alpha\left\langle\mathbf{Q}^{T} \mathbf{A}_{r i} \mathbf{Q}, \mathbf{A}_{r}^{0}\right\rangle+\langle\mathbf{F}, \mathbf{Q}\rangle+\left\langle\frac{1}{2} \mathbf{S}, \mathbf{I}_{k}-\mathbf{Q}^{T} \mathbf{Q}\right\rangle,
\end{aligned}
$$

where $\frac{1}{2} \mathbf{S} \in \mathbb{R}^{k \times k}$ is a symmetric matrix of Lagrangian multipliers. Using the following identities [21],

$$
\begin{aligned}
\nabla_{\mathbf{Q}}\langle\mathbf{M}, \mathbf{Q}\rangle & =\mathbf{M} \\
\nabla_{\mathbf{Q}}\left\langle\mathbf{M}, \mathbf{Q}^{T} \mathbf{Q}\right\rangle & =\mathbf{Q}\left(\mathbf{M}+\mathbf{M}^{T}\right) \\
\nabla_{\mathbf{Q}}\left\langle\mathbf{Q}^{T} \mathbf{M} \mathbf{Q}, \mathbf{N}\right\rangle & =\mathbf{M Q N}^{T}+\mathbf{M}^{T} \mathbf{Q N}
\end{aligned}
$$

the gradient of the Lagrangian with respect to $\mathbf{Q}$ is obtained as

$$
\nabla_{\mathbf{Q}} \mathcal{L}(\mathbf{Q}, \mathbf{S})=\epsilon\left(\mathbf{E}_{r i} \mathbf{Q}\left(\mathbf{E}_{r}^{0}\right)^{T}+\mathbf{E}_{r i}^{T} \mathbf{Q} \mathbf{E}_{r}^{0}\right)+\alpha\left(\mathbf{A}_{r i} \mathbf{Q}\left(\mathbf{A}_{r}^{0}\right)^{T}+\mathbf{A}_{r i}^{T} \mathbf{Q} \mathbf{A}_{r}^{0}\right)+\mathbf{F}-\mathbf{Q S}
$$

which leads to the first-order optimality condition

$$
\mathbf{Q S}=\epsilon\left(\mathbf{E}_{r i} \mathbf{Q}\left(\mathbf{E}_{r}^{0}\right)^{T}+\mathbf{E}_{r i}^{T} \mathbf{Q} \mathbf{E}_{r}^{0}\right)+\alpha\left(\mathbf{A}_{r i} \mathbf{Q}\left(\mathbf{A}_{r}^{0}\right)^{T}+\mathbf{A}_{r i}^{T} \mathbf{Q} \mathbf{A}_{r}^{0}\right)+\mathbf{F},
$$

together with the constraint $\mathbf{Q}^{T} \mathbf{Q}=\mathbf{I}_{k}$ and the property that $\mathbf{S}$ is symmetric. 


\section{Appendix 2: proof of Theorem 2}

Here, the goal is to prove that the set of fixed points of the proposed recursive algorithm is the same as the set of stationary points of the objective function $\mathcal{J}_{G}$.

Let $\widehat{\mathbf{Q}}$ denote a fixed point of the recursive method defined in Algorithm 2. Then, $\widehat{\mathbf{Q}}$ satisfies $\widehat{\mathbf{Q}}=\widehat{\mathbf{U}} \widehat{\mathbf{V}}^{T}$, where

$$
\widehat{\mathbf{U}} \widehat{\boldsymbol{\Sigma}} \widehat{\mathbf{V}}^{T}=\epsilon\left(\mathbf{E}_{r i} \widehat{\mathbf{Q}}\left(\mathbf{E}_{r}^{0}\right)^{T}+\mathbf{E}_{r i}^{T} \widehat{\mathbf{Q}} \mathbf{E}_{r}^{0}\right)+\alpha\left(\mathbf{A}_{r i} \widehat{\mathbf{Q}}\left(\mathbf{A}_{r}^{0}\right)^{T}+\mathbf{A}_{r i}^{T} \widehat{\mathbf{Q}} \mathbf{A}_{r}^{0}\right)+s \widehat{\mathbf{Q}}+\mathbf{F}
$$

is a singular value decomposition. Since $\widehat{\mathbf{V}}$ is an orthogonal matrix,

$$
\widehat{\mathbf{U}} \widehat{\mathbf{V}}^{T} \widehat{\mathbf{V}} \widehat{\boldsymbol{\Sigma}} \widehat{\mathbf{V}}^{T}=\epsilon\left(\mathbf{E}_{r i} \widehat{\mathbf{Q}}\left(\mathbf{E}_{r}^{0}\right)^{T}+\mathbf{E}_{r i}^{T} \widehat{\mathbf{Q}} \mathbf{E}_{r}^{0}\right)+\alpha\left(\mathbf{A}_{r i} \widehat{\mathbf{Q}}\left(\mathbf{A}_{r}^{0}\right)^{T}+\mathbf{A}_{r i}^{T} \widehat{\mathbf{Q}} \mathbf{A}_{r}^{0}\right)+s \widehat{\mathbf{Q}}+\mathbf{F},
$$

that is,

$$
\widehat{\mathbf{Q}} \mathbf{S}=\epsilon\left(\mathbf{E}_{r i} \widehat{\mathbf{Q}}\left(\mathbf{E}_{r}^{0}\right)^{T}+\mathbf{E}_{r i}^{T} \widehat{\mathbf{Q}} \mathbf{E}_{r}^{0}\right)+\alpha\left(\mathbf{A}_{r i} \widehat{\mathbf{Q}}\left(\mathbf{A}_{r}^{0}\right)^{T}+\mathbf{A}_{r i}^{T} \widehat{\mathbf{Q}} \mathbf{A}_{r}^{0}\right)+\mathbf{F}
$$

where $\mathbf{S}=\widehat{\mathbf{V}} \widehat{\mathbf{\Sigma}} \widehat{\mathbf{V}}^{T}-s \mathbf{I}_{k}$ is a symmetric matrix. Since $\widehat{\mathbf{U}}$ and $\widehat{\mathbf{V}}$ are orthogonal, it follows that $\widehat{\mathbf{Q}}$ is also orthogonal and therefore meets the requirements of Theorem 2. Hence, the set of fixed points of Algorithm 2 is included in the set of stationary points of $\mathcal{J}_{G}$.

Conversely, let $\mathbf{Q}^{\star}$ be a stationary point of $\mathcal{J}_{G}$. Then, there exists a symmetric matrix $\mathbf{S}$ such that Eq. (10) holds with $\mathbf{Q}^{\star}$ orthogonal. Since $\mathbf{S}$ is real and symmetric, it is diagonalizable and therefore can be written as

$$
\mathbf{S}=\mathbf{U} \boldsymbol{\Lambda} \mathbf{U}^{T}
$$

where the eigenvalues in $\boldsymbol{\Lambda}$ are real and ordered decreasingly, and $\mathbf{U}$ is an orthogonal matrix. Then,

$$
\mathbf{Q}^{\star} \mathbf{U} \mathbf{\Lambda} \mathbf{U}^{T}+s \mathbf{Q}^{\star}=\epsilon\left(\mathbf{E}_{r i} \mathbf{Q}^{\star}\left(\mathbf{E}_{r}^{0}\right)^{T}+\mathbf{E}_{r i}^{T} \mathbf{Q}^{\star} \mathbf{E}_{r}^{0}\right)+\alpha\left(\mathbf{A}_{r i} \mathbf{Q}^{\star}\left(\mathbf{A}_{r}^{0}\right)^{T}+\mathbf{A}_{r i}^{T} \mathbf{Q}^{\star} \mathbf{A}_{r}^{0}\right)+\mathbf{F}+s \mathbf{Q}^{\star},
$$

which can also be written as

$$
\left(\mathbf{Q}^{\star} \mathbf{U}\right)(\boldsymbol{\Lambda}+s \mathbf{I}) \mathbf{U}^{T}=\epsilon\left(\mathbf{E}_{r i} \mathbf{Q}^{\star}\left(\mathbf{E}_{r}^{0}\right)^{T}+\mathbf{E}_{r i}^{T} \mathbf{Q}^{\star} \mathbf{E}_{r}^{0}\right)+\alpha\left(\mathbf{A}_{r i} \mathbf{Q}^{\star}\left(\mathbf{A}_{r}^{0}\right)^{T}+\mathbf{A}_{r i}^{T} \mathbf{Q}^{\star} \mathbf{A}_{r}^{0}\right)+s \mathbf{Q}^{\star}+\mathbf{F} .
$$

In order to conclude the proof, it remains to show that $\left(\mathbf{Q}^{\star} \mathbf{U}\right)(\boldsymbol{\Lambda}+s \mathbf{I}) \mathbf{U}^{T}$ is a singular value decomposition. To this effect, note that $\mathbf{Q}^{\star} \mathbf{U}$ and $\mathbf{U}$ are orthogonal matrices and $\boldsymbol{\Lambda}+s \mathbf{I}$ is a diagonal matrix, hence it suffices to show that $\boldsymbol{\Lambda}+s \mathbf{I}$ has all diagonal entries positive.

Now, from Eq. (19), it follows that

$$
\begin{aligned}
\|\boldsymbol{\Lambda}\|_{2}= & \left\|\mathbf{Q}^{\star} \mathbf{U} \mathbf{\Lambda} \mathbf{U}^{T}\right\|_{2}=\left\|\epsilon\left(\mathbf{E}_{r i} \mathbf{Q}^{\star}\left(\mathbf{E}_{r}^{0}\right)^{T}+\mathbf{E}_{r i}^{T} \mathbf{Q}^{\star} \mathbf{E}_{r}^{0}\right)+\alpha\left(\mathbf{A}_{r i} \mathbf{Q}^{\star}\left(\mathbf{A}_{r}^{0}\right)^{T}+\mathbf{A}_{r i}^{T} \mathbf{Q}^{\star} \mathbf{A}_{r}^{0}\right)+\mathbf{F}\right\|_{2} \\
\leq & \epsilon\left\|\mathbf{E}_{r i} \mathbf{Q}^{\star}\left(\mathbf{E}_{r}^{0}\right)^{T}+\mathbf{E}_{r i}^{T} \mathbf{Q}^{\star} \mathbf{E}_{r}^{0}\right\|_{2}+\alpha\left\|\mathbf{A}_{r i} \mathbf{Q}^{\star}\left(\mathbf{A}_{r}^{0}\right)^{T}+\mathbf{A}_{r i}^{T} \mathbf{Q}^{\star} \mathbf{A}_{r}^{0}\right\|_{2}+\|\mathbf{F}\|_{2} \\
\leq & \epsilon\left(\left\|\mathbf{E}_{r i}\right\|_{2}\left\|\mathbf{Q}^{\star}\right\|_{2}\left\|\left(\mathbf{E}_{r}^{0}\right)^{T}\right\|_{2}+\left\|\mathbf{E}_{r i}^{T}\right\|_{2}\left\|\mathbf{Q}^{\star}\right\|_{2}\left\|\mathbf{E}_{r}^{0}\right\|_{2}\right) \\
& \quad+\alpha\left(\left\|\mathbf{A}_{r i}\right\|_{2}\left\|\mathbf{Q}^{\star}\right\|_{2}\left\|\left(\mathbf{A}_{r}^{0}\right)^{T}\right\|_{2}+\left\|\mathbf{A}_{r i}^{T}\right\|_{2}\left\|\mathbf{Q}^{\star}\right\|_{2}\left\|\mathbf{A}_{r}^{0}\right\|_{2}\right)+\|\mathbf{F}\|_{2} \\
& \leq 2 \epsilon\left\|\mathbf{E}_{r i}\right\|\left\|_{2}\right\| \mathbf{E}_{r}^{0}\left\|_{2}+2 \alpha\right\| \mathbf{A}_{r i}\left\|_{2}\right\| \mathbf{A}_{r}^{0}\left\|_{2}+\right\| \mathbf{F} \|_{2} \\
\leq & s_{\min , \mathrm{G}}
\end{aligned}
$$

by definition of $s_{\text {min,G }}$ in Eq. (9). Denoting by $\lambda_{i} i=1, \cdots, k$ the diagonal entries in $\boldsymbol{\Lambda}$, this implies that

$$
-s_{\min , \mathrm{G}} \leq \lambda_{i} \leq s_{\min , G}, i=1, \cdots, k,
$$

and since $s>s_{\min , \mathrm{G}}$,

$$
\lambda_{i}+s>0, i=1, \cdots, k .
$$

Hence, the set of stationary points of $\mathcal{J}_{G}$ is included in the set of fixed points of Algorithm 2. Therefore, the two sets are identical, which concludes the proof. 


\section{References}

[1] B. Moore, Principal component analysis in linear systems: Controllability, observability, and model reduction, IEEE Transactions on Automatic Control 26 (1981) 17-32.

[2] L. Sirovich, Turbulence and the dynamics of coherent structures. Part I: coherent structures, Quarterly of Applied Mathematics 45 (1987) 561-571.

[3] D. Amsallem, M. J. Zahr, C. Farhat, Nonlinear model order reduction based on local reduced-order bases, International Journal for Numerical Methods in Engineering 92 (2012) 891-916.

[4] D. Ryckelynck, A priori hyperreduction method: an adaptive approach, Journal of Computational Physics 202 (2005) 346-366.

[5] S. Chaturantabut, D. Sorensen, Nonlinear model reduction via discrete empirical interpolation, SIAM Journal on Scientific Computing 32 (2010) 2737-2764.

[6] K. Carlberg, C. Bou-Mosleh, C. Farhat, Efficient non-linear model reduction via a least-squares PetrovGalerkin projection and compressive tensor approximations, International Journal for Numerical Methods in Engineering 86 (2011) 155-181.

[7] C. Farhat, T. Chapman, P. Avery, Stability and accuracy properties of the energy-conserving sampling and weighting (ECSW) method for the hyper reduction of nonlinear finite element dynamic models, International Journal for Numerical Methods in Engineering 102 (2015) 1077-1110.

[8] K. Carlberg, C. Farhat, J. Cortial, D. Amsallem, The GNAT method for nonlinear model reduction: effective implementation and application to computational fluid dynamics and turbulent flows, Journal of Computational Physics 242 (2013) 623-647.

[9] C. Farhat, P. Avery, T. Chapman, J. Cortial, Dimensional reduction of nonlinear finite element dynamic models with finite rotations and energy-based mesh sampling and weighting for computational efficiency, International Journal for Numerical Methods in Engineering 98 (2014) 625-662.

[10] K. Veroy, A. T. Patera, Certified real-time solution of the parametrized steady incompressible NavierStokes equations: rigorous reduced-basis a posteriori error bounds, International Journal for Numerical Methods in Fluids 47 (2005) 773-788.

[11] T. Lieu, C. Farhat, M. Lesoinne, Reduced-order fluid/structure modeling of a complete aircraft configuration, Computer Methods in Applied Mechanics and Engineering 195 (2006) 5730-5742.

[12] D. Amsallem, C. Farhat, Interpolation method for adapting reduced-order models and application to aeroelasticity, AIAA Journal 46 (2008) 1803-1813.

[13] D. Amsallem, J. Cortial, C. Farhat, Toward real-time computational-fluid-dynamics-based aeroelastic computations using a database of reduced-order information, AIAA Journal 48 (2010) 2029-2037.

[14] D. Amsallem, Interpolation on Manifolds of CFD-Based Fluid and Finite Element-Based Structural Reduced-Order Models for On-Line Aeroelastic Predictions, Ph.D. thesis, Stanford University, 2010.

[15] J. Degroote, J. Vierendeels, K. Willcox, Interpolation among reduced-order matrices to obtain parameterized models for design, optimization and probabilistic analysis, International Journal for Numerical Methods in Fluids 63 (2010) 207-230.

[16] H. Panzer, J. Mohring, R. Eid, B. Lohmann, Parametric model order reduction by matrix interpolation, at-Automatisierungstechnik 58 (2010) 475-484.

[17] D. Amsallem, C. Farhat, An online method for interpolating linear parametric reduced-order models, SIAM Journal on Scientific Computing 33 (2011) 2169-2198. 
[18] G. Berkooz, P. Holmes, J. L. Lumley, The proper orthogonal decomposition in the analysis of turbulent flows, Annual Review of Fluid Mechanics 25 (1993) 539-575.

[19] E. J. Grimme, Krylov Projection Methods for Model Reduction, Ph.D. thesis, University of Illinois at Urbana Champaign, 1997.

[20] U. Hetmaniuk, R. Tezaur, C. Farhat, Review and assessment of interpolatory model order reduction methods for frequency response structural dynamics and acoustics problems, International Journal for Numerical Methods in Engineering 90 (2012) 1636-1662.

[21] C. Fraikin, Y. Nesterov, P. V. Dooren, Optimizing the coupling between two isometric projections of matrices, SIAM Journal on Matrix Analysis and Applications 30 (2008) 324-345.

[22] U. Helmke, J. Barratt Moore, Optimization and Dynamical Systems, Springer, 1994.

[23] M. A. Grepl, A. T. Patera, A posteriori error bounds for reduced-basis approximations of parametrized parabolic partial differential equations, ESAIM: Mathematical Modelling and Numerical Analysis 39 (2005) 157-181.

[24] T. Bui-Thanh, K. Willcox, O. Ghattas, Parametric reduced-order models for probabilistic analysis of unsteady aerodynamic applications, AIAA Journal 46 (2008) 2520-2529.

[25] R. Zimmermann, A locally parametrized reduced-order model for the linear frequency domain approach to time-accurate computational fluid dynamics, SIAM Journal on Scientific Computing 36 (2014) B508B537.

[26] D. Colton, R. Kress, Inverse Acoustic and Electromagnetic Scattering Theory, Springer, 2013.

[27] J.-P. Berenger, A perfectly matched layer for the absorption of electromagnetic waves, Journal of Computational Physics 114 (1994) 185-200.

[28] C. Farhat, R. Tezaur, R. Djellouli, On the solution of three-dimensional inverse obstacle acoustic scattering problems by a regularized Newton method, Inverse Problems 18 (2002) 1229-1246.

[29] U. Hetmaniuk, R. Tezaur, C. Farhat, An adaptive scheme for a class of interpolatory model reduction methods for frequency response problems, International Journal for Numerical Methods in Engineering 93 (2013) 1109-1124.

[30] B. Haasdonk, M. Dihlmann, M. Ohlberger, A training set and multiple bases generation approach for parameterized model reduction based on adaptive grids in parameter space, Math. Comput. Model. Dyn. Syst. 17 (2011) 423-442.

[31] J. L. Eftang, A. T. Patera, E. M. Rønquist, An " $h p$ " certified reduced basis method for parametrized elliptic partial differential equations, SIAM J. Sci. Comput. 32 (2010) 3170-3200.

[32] C. Farhat, E. K.-y. Chiu, D. Amsallem, J.-S. Schotté, R. Ohayon, Modeling of fuel sloshing and its physical effects on flutter, AIAA Journal (2013) 1-14.

[33] M. Lesoinne, M. Sarkis, U. Hetmaniuk, C. Farhat, A CFD based method for solving aeroelastic eigenproblems in the subsonic, transonic, and supersonic regimes, Computer Methods in Applied Mechanics and Engineering 190 (2001) 3121-3146.

[34] E. C. Yates, AGARD Standard Aeroelastic Configurations For Dynamic Response - 1 - Wing 445.6, NASA, 1987. 